\title{
Drosophila Mmp2 Regulates the Matrix Molecule Faulty Attraction (Frac) to Promote Motor Axon Targeting in Drosophila
}

\author{
Crystal M. Miller, ${ }^{1}$ Nan Liu, ${ }^{1}$ Andrea Page-McCaw, ${ }^{2}$ and Heather T. Broihier ${ }^{1}$ \\ ${ }^{1}$ Department of Neurosciences, Case Western Reserve University School of Medicine, Cleveland, Ohio 44106, and ${ }^{2}$ Department of Cell and Developmental \\ Biology, Vanderbilt University Medical Center, Nashville, Tennessee 37232
}

\begin{abstract}
Matrix metalloproteinases (MMPs) are widely hypothesized to regulate signaling events through processing of extracellular matrix (ECM) molecules. We previously demonstrated that membrane-associated Mmp2 is expressed in exit glia and contributes to motor axon targeting. To identify possible substrates, we undertook a yeast interaction screen for Mmp2-binding proteins and identified the novel ECM protein faulty attraction (Frac). Frac encodes a multidomain extracellular protein rich in epidermal growth factor (EGF) and calcium-binding EGF domains, related to the vertebrate Fibrillin and Fibulin gene families. It is expressed in mesodermal domains flanking Mmp2-positive glia. The juxtaposition of Mmp2 and Frac proteins raises the possibility that Frac is a proteolytic target of Mmp2. Consistent with this hypothesis, levels of full-length Frac are increased in Mmp2 loss-of-function (LOF) and decreased in Mmp2 gain-offunction (GOF) embryos, indicating that Frac cleavage is Mmp2 dependent. To test whether frac is necessary for axon targeting, we characterized guidance in frac LOF mutants. Motor axons in frac LOF embryos are loosely associated and project ectopically, a phenotype essentially equivalent to that of $M m p 2$ LOF. The phenotypic similarity between enzyme and substrate mutants argues that Mmp2 activates Frac. In addition, Mmp2 overexpression pathfinding phenotypes depend on frac activity, indicating that Mmp2 is genetically upstream of frac. Last, overexpression experiments suggest that Frac is unlikely to have intrinsic signaling activity, raising the possibility that an Mmp2-generated Frac fragment acts as a guidance cue cofactor. Indeed, we present genetic evidence that Frac regulates a non-canonical LIM kinase 1-dependent bone morphogenetic protein signaling pathway in motoneurons necessary for axon pathfinding during embryogenesis.
\end{abstract}

\section{Introduction}

The mechanisms that confer spatial and temporal precision to signal transduction by axon guidance receptors remain elusive. Although substantial progress has been made in identifying proteins required for axon pathfinding (Dickson, 2002), the regulatory systems that modulate their activity to give rise to stereotyped axonal trajectories are unclear. Localized proteolysis of receptors or their ligands is an attractive mechanism to contribute to the spatiotemporal specificity of axon pathfinding (Meyer and Aberle, 2006; Serpe and O'Connor, 2006; Miller et al., 2008).

The genetic advantages Drosophila affords have proven essential to the characterization of conserved pathways and proteins. Matrix metalloproteinase (MMP) family members can degrade structural components of the extracellular matrix (ECM) and are of substantial clinical interest, because they are often upregulated

Received Sept. 13, 2010; revised Jan. 28, 2011; accepted Feb. 1, 2011.

This work was supported by National Institutes of Health Grants R01NS055245 (H.T.B.), F31NS063661 (C.M.M.), and R01GM073883 (A.P.-M.) and the Mt. Sinai Foundation (H.T.B.). We thank Sarah Forcier, Bernadette Glasheen, Julia Serrano, and Chris Dejelo for excellent technical assistance. We are grateful to the members of the Broihier laboratory for thoughtful discussions and practical help.

Correspondence should be addressed to Heather T. Broihier, Department of Neurosciences, Case Western Reserve University School of Medicine, 10900 Euclid Avenue, Cleveland, 0H 44106. E-mail: heather.broihier@case.edu.

DOI:10.1523/JNEUROSCI.4811-10.2011

Copyright $\odot 2011$ the authors $\quad 0270-6474 / 11 / 315335-13 \$ 15.00 / 0$ in inflammatory and metastatic contexts. However, their nonpathological actions, and in particular the identities of their substrates, remain poorly defined (Page-McCaw et al., 2007; Kessenbrock et al., 2010; Rodríguez et al., 2010). Relevant to the work presented here, compensation and redundancy among the 24 vertebrate MMP family members have necessitated in vitro approaches to characterizing their functions. Drosophila provides a greatly simplified in vivo paradigm for the characterization of MMP function, because the fly genome contains only two MMP homologs: Mmp1, a secreted protein, and Mmp2, which is membrane associated (Llano et al., 2000, 2002; Page-McCaw et al., 2003). An analysis of Drosophila MMP mutants demonstrated that MMPs are required for proper motor axon pathfinding. In particular, Mmp2 is expressed in motor axon-associated exit glia and promotes axon fasciculation (Miller et al., 2008). It is reasonable to consider that the Drosophila system could also facilitate MMP substrate identification.

Here, we demonstrate that faulty attraction ( frac) is an Mmp2 substrate essential for motor axon targeting. Frac was identified as a potential Mmp2 substrate in a yeast interaction screen. It is predicted to encode an ECM protein containing EGF and calcium-binding EGF (cbEGF) repeats, related to the vertebrate Fibrillins and Fibulins (Downing et al., 1996). Fibrillins are large matrix-associated glycoproteins that comprise the major structural protein of microfibrils (Sakai et al., 1986; Zhang et al., 1995). 
In addition to giving structural support to connective tissue, they are important regulators of TGF $\beta$ bioavailability (Charbonneau et al., 2004; Dietz et al., 2005).

We provide evidence that Frac is an Mmp2 substrate via biochemical, expression, and genetic analyses. Frac is expressed in mesoderm flanking extending axons and immediately adjacent to Mmp2-positive glia. Our data indicate that Mmp2 cleaves and activates Frac to promote motor axon bundling during outgrowth. We argue that Mmp2 and Frac act in a common pathway, because (1) Mmp2 and frac loss-of-function (LOF) mutants are indistinguishable from each other, and (2) the phenotype of frac LOF mutants is epistatic to that of $M m p 2$ gain-of-function (GOF) mutants, arguing that $M m p 2$ is genetically upstream of frac. The close functional relationship between mammalian Fibrillins and TGF $\beta$ s prompted us to probe for a link between frac and the bone morphogenetic protein (BMP) pathway. We find that frac likely regulates the activation of a non-canonical LIM kinase 1 (LIMK1)-dependent pathway in motoneurons.

\section{Materials and Methods}

Fly stocks. Stocks used in this work include the following: $M m p 2^{W 307 *}$, $M m p 1^{\text {Q112*}}$, UAS-Mmp1, UAS-Mmp2, tubulinGAL4 (Page-McCaw et al., 2003), viking::GFP (a kind gift from A. Prokop, The University of Manchester, Manchester, UK), UAS-sax ${ }^{a c t}$ (a kind gift from K. Wharton, Brown University, Providence, RI), $\mathrm{frac}^{\Delta 1}$ (see below), and $\mathrm{frac}^{\Delta 2}$ (see below). UAS-frac was generated from a full-length cDNA plasmid template (RE24628), cloned into pUAST, and injected into flies by standard methods. Similar results were observed with at least two independent transgenic lines, UAS-frac \#3 and UAS-frac \#7, to control for position and copy number effects. elavGAL4 is either from A. DiAntonio (Washington University, St. Louis, MO) or C155GAL4 on the X chromosome available from the Bloomington Stock Center. RNA interference (RNAi) lines are from the Exelixis collection and were analyzed in combination with UAS-Dicer2 to augment gene knockdown. All remaining stocks were obtained from the Bloomington Stock Center. Double mutants were generated using standard genetic techniques.

Minos element excision. Minos imprecise excision was performed as described (Metaxakis et al., 2005). Briefly, flies heterozygous for a single Minos transposon insertion, Mi\{ET1 $\}$ CG7526 ${ }^{\text {MB05690 }}$ [marked by enhanced green fluorescent protein (EGFP)] in the first intron of the frac gene region were crossed to flies carrying the Minos transposase (marked by white). Two days after setting up the cross, adults were transferred to new vials, and the old vials were heat shocked daily for $1 \mathrm{~h}$ in a $37^{\circ}$ water bath until pupariation. Adults carrying both the transposon and transposase were then crossed to a chromosome 3 balancer stock. Progeny with transposon excisions were identified as carrying the correct balancers but lacking EGFP and white markers. These flies were crossed individually to a chromosome 3 balancer and analyzed for imprecise excision events using PCR.

Immunohistochemistry. Embryo fixation, antibody staining, and RNA in situ hybridization were performed as described (Miller et al., 2008). The following primary antibodies were used: mAb1D4 [Fasciclin II (FasII)] at 1:10 [generated by C. Goodman (University of California, Berkeley, CA) and obtained from the Developmental Studies Hybridoma Bank (DSHB)], monoclonal antibody (mAb) myosin heavy chain (MHC) at 1:500 (a kind gift from Erika Geisbrecht, University of Missouri-Kansas City, Kansas City, MO), guinea pig antibody (gpAb) Kakapo/Shortstop at 1:300 (a kind gift from Talila Volk, Weizman Institute of Science, Rehovot, Israel), mAb repo at 1:10 (generated by C. Goodman and obtained from the DSHB), rabbit antibody (rbAb) pMad/ pSmad3 (Epitomics) at 1:100, and rbAb $\beta$-galactosidase (Immunology Laboratory Consultants) at 1:1000. For RNA in situ hybridization, the digoxigenin (DIG)-labeled probe was made using the Roche DIG RNA Labeling kit (SP6/T7) using the full-length Frac cDNA plasmid as the template. Guinea pig and rabbit anti-Frac antibodies were generated against a peptide composed of residues 1216-1500 of the Frac protein. Antibodies generated in different animals from both species all recognized the same protein expression pattern on whole-mount embryos. For immunohistochemistry, Frac antibodies were affinity purified using the AmminoLink Plus Immobilization kit (Pierce) and were not diluted for use on embryos. All microscopy was performed as described (Miller et al., 2008). Data analysis was performed using Fisher's exact test unless otherwise stated.

Western blotting. Western blot protein samples were prepared by boiling 60 embryos (stage 15-17) in $36 \mu$ l of Laemli's sample buffer. A total of $25 \mu \mathrm{l}$ of lysate, equivalent to 42 embryos, was loaded into each lane. Protein was transferred to Millipore Immobilon-P $0.45 \mu \mathrm{M}$ membrane. gpAb Frac whole serum was used at a 1:100 dilution, and goat polyclonal antibody glyceraldehyde-3-phosphate dehydrogenase (GAPDH) (Imgenex) was used as a loading control at 1:5000. HRP-labeled goat antiguinea pig and rabbit anti-goat (MP Biomedicals) were diluted at 1:10,000. Bands were detected using the Supersignal West Pico Chemiluminescent Substrate kit (Thermo Fisher Scientific). Band intensities were quantified using NIH ImageJ 1.43 software. Statistical significance was calculated using single-factor ANOVA.

Yeast two-hybrid screen. The catalytically inactive Mmp2 plasmid in which E258 was mutated to A was engineered by splice overlap extension (Vallejo et al., 1994) from an Mmp2 cDNA (Page-McCaw et al., 2003). The bait plasmid pGBD-C1.Mmp2CAT was constructed by inserting catalytically inactive Mmp2 into pGBD-C1 (James et al., 1996). We screened $1.8 \times 10^{5}$ cDNAs from a Drosophila embryo library and $2.3 \times$ $10^{5}$ cDNAs from a third-instar larval library (Durfee et al., 1993) using the methods of James et al. (1996) as described (Zhang et al., 2006). Catalytically inactive Mmp2 positively interacted with six predicted extracellular or transmembrane proteins, including the hyalin domain region of Frac. A parallel screen for Mmp1 interactors successfully identified Ninjurin A as an in vivo substrate for Mmp1 illustrating the effectiveness of this method (Zhang et al., 2006).

\section{Results}

\section{CG7526 interacts with Mmp2 and is related to vertebrate cbEGF-domain-containing proteins}

We previously demonstrated that the catalytic activity of Mmp2 promotes motor axon fasciculation (Miller et al., 2008), implying that its substrate also functions in axon targeting. As a route to substrate identification, we performed a yeast interaction screen using a substrate-trapping E258A mutant of Mmp2 as bait (see Materials and Methods). The screen yielded six transmembrane or extracellular proteins representing potential substrates. We examined their RNA expression profiles via in situ hybridization to determine whether any were expressed in a manner consistent with regulation by Mmp2. Mmp2 is predicted to have a glycosylphosphatidylinositol (GPI) anchor (Llano et al., 2000, 2002; Page-McCaw et al., 2003) and is expressed by exit glia, which are tightly associated with motor axons (Sepp et al., 2001; Sepp and Auld, 2003; Freeman, 2006; Miller et al., 2008). Hence, we anticipated relevant substrates would be expressed in a pattern consistent with cleavage by exit glia-associated Mmp2, namely the glia themselves, neurons, or mesoderm. We identified one gene, CG7526, with salient mesodermal expression (see below and Fig. 2).

Sequence analysis indicates that CG7526 codes for a predicted extracellular protein, including three EGF and seven cbEGF repeats (Fig. 1A). CG7526 shares a conserved domain structure with extracellular proteins containing arrays of cbEGF repeats (Fig. 1A). Calcium binding is thought to impart structural rigidity to these proteins, and the cbEGF domains also participate in protein-protein interactions (Handford et al., 1995; Downing et al., 1996). Best characterized among related vertebrate proteins are Fibrillins, which serve both structural and signaling functions in the ECM. Fibrillins are modular glycoproteins that compose microfibrils and thus function as structural components of the ECM (Sakai et al., 1986; Zhang et al., 1995). They are characterized by arrays of cbEGF domains interspersed by TGF $\beta$-binding (TB) domains. In addition to their structural role, Fibrillins reg- 


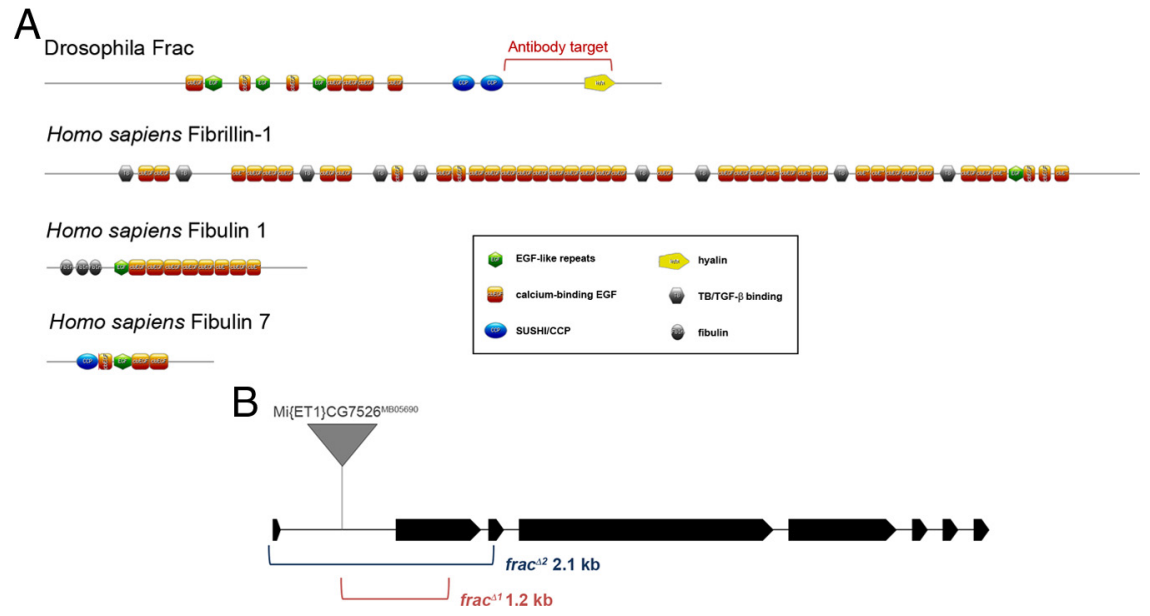

Figure 1. frac gene structure and allele generation. $\boldsymbol{A}$, Domain structure of Drosophila Frac and Homo sapiens Fibrillin-1, Fibulin 1, and Fibulin 7. Two Fibulins were included to account for the variation between family members. Domains pictured include EGF-like repeats (green), cbEGF (orange), CCP/SUSHI (blue), hyalin (yellow pentagon), TB (gray hexagon), and fibulin (gray circles). $\boldsymbol{B}$, Schematic of the frac gene region and span of frac LOF alleles. Exons are represented by rectangular black boxes and introns by thin lines. Position of Minos insertion is displayed.

ulate TGF $\beta$ superfamily bioavailability (Charbonneau et al., 2004; Ramirez and Rifkin, 2009). Underscoring the major functional relationship between Fibrillins and TGF $\beta$ signaling, human Marfan syndrome (MFS) results from haploinsufficiency of Fibrillin-1 and is strongly linked to elevated levels of active TGF $\beta$ (Ramirez and Dietz, 2007). Although CG7526 is structurally related to Fibrillins, the presence of CCP and hyalin domains, coupled with the absence of TB domains, argues that it is not orthologous (Hynes and Zhao, 2000). Based on the axon guidance phenotype described here, we have named CG7526 faulty attraction ( frac).

\section{Drosophila Frac is expressed in the embryonic mesoderm}

We characterized the expression profile of frac to ask whether it is expressed in a pattern consistent with regulation by Mmp2. At embryonic stage 13, frac RNA is expressed strongly in ventral mesoderm adjacent to the CNS and at lower levels in more dorsal mesoderm (Fig. 2A). At stage 15, the frac RNA expression domain expands to include all embryonic musculature (Fig. 2 B). To characterize Frac protein expression, we generated anti-Frac antibodies (Fig. 1A). Confirming antibody specificity, anti-Frac antibodies do not recognize protein in $\mathrm{frac}^{\Delta 1}$ mutant embryos (see Fig. $4 A, B)$. The dynamic pattern of frac RNA expression in the mesoderm is recapitulated by Frac protein. At stage 14, Frac expression is apparent in wedges of ventral mesoderm immediately adjacent to the nerve cord and then expands to encompass all embryonic muscle fibers at stage 15 (Fig. 2C,D). Frac continues to be expressed throughout the embryonic musculature until late stage 17, when its expression concentrates to slivers at hemisegment boundaries in a pattern characteristic of muscle attachment sites (Fig. 2E) (Becker et al., 1997). Confirming that Frac is mesodermal, it is coexpressed with myosin heavy chain, a wellcharacterized muscle marker (Fig. 1F) (Kiehart and Feghali, 1986). Furthermore, we verified that Frac expression in late stage 17 embryos is restricted to muscle attachment sites by double labeling with Shortstop/Kakapo (Fig. 2G) (Strumpf and Volk, 1998). Because the wedges of Frac-positive ventral mesoderm appear to be in close proximity to Mmp2-positive exit glia, Frac may represent an Mmp2 substrate. Hence, we analyzed the relative positions of Frac protein, Mmp2-positive glia, and motor axons in more detail. To compare the position of Frac protein to extending motor axons, we double-labeled wild-type embryos with anti-Frac antibodies and antiFasII antibodies, which marks motor projections (Van Vactor et al., 1993). Motor axons extend through the periphery at stage 14, a time when high levels of Frac are expressed in the muscles through which these nerves traverse (Fig. $2 \mathrm{H}$ ). Because Mmp2 is expressed by exit glia and is membrane bound (Llano et al., 2002; Miller et al., 2008), if mesodermal Frac is an in vivo substrate of Mmp2, it is predicted to be present immediately adjacent to these glia. In support of this hypothesis, Repo-positive glia align along the edge of the Frac-positive wedge of ventral mesoderm (arrows point to exit glia in Fig. 2I). This striking juxtaposition of Frac-positive mesoderm and Mmp2-positive glia argues that Mmp2 is poised to cleave Frac during embryogenesis. To examine the relative positions of Frac-positive mesoderm and glial processes in the periphery at later embryonic stages, we colabeled repo $>$ actin-GFP embryos with anti-Frac and anti-GFP. Glial cell processes extend through Frac-expressing mesoderm (Fig. $2 \mathrm{~J}$ ), indicating that membrane-tethered Mmp2 is appropriately positioned to cleave Frac.

\section{Frac is processed in an Mmp2-dependent manner}

Frac was identified in a yeast two-hybrid screen as an Mmp2 interactor, and its embryonic expression domain is in line with regulation by Mmp2. Hence, we investigated whether Frac represents a proteolytic substrate of Mmp2. Frac has a predicted size of $172 \mathrm{kDa}$. We find that Frac runs as a doublet at 170 and 250 $\mathrm{kDa}$ on Western blot of wild-type embryo extracts (Fig. $3 A$ ). The larger molecular weight band likely reflects a posttranslationally modified form of Frac, because many matrix molecules are heavily glycosylated (Comer and Hart, 2000). These two bands are not present in extracts from frac deletion embryos, demonstrating specificity (Fig. 3A). To determine whether Mmp2 is responsible for processing Frac, we analyzed Frac expression in $M m p 2 \mathrm{LOF}$ and GOF embryo extracts (Fig. $3 A$ ). In $M m p 2$ misexpression embryos $(t u b>M m p 2)$, full-length Frac is nearly absent, arguing that Mmp2 can process the full-length form. Consistent with this model, three small molecular weight Frac fragments are observed in extracts from Mmp2 misexpression embryos (arrowheads). Conversely, in $M m p 2^{W 307 *}$ embryo extracts, we do not observe the small molecular weight bands and we consistently detect a significant increase in the amount of full-length Frac (Fig. $3 A, B$ ), arguing that Frac is not appropriately processed in the absence of Mmp2. The alterations in Frac processing observed in $M m p 2$ mutant embryonic extracts provide evidence that Frac is sensitive to Mmp2 levels.

The marked reduction in full-length Frac in Mmp2 GOF protein extracts indicated that we might detect a visible reduction in Frac expression in these embryos if Frac is processed by Mmp2 and the cleavage fragments are degraded. In stage 14 wild-type embryos, Frac is expressed strongly in wedges of ventral mesoderm. We compared Frac levels in Mmp2 GOF embryos and wild-type embryos at stage 14, with the observer blinded to genotype. We found a 2.5-fold reduction in fluorescence intensity in 
embryos with Mmp2 misexpression relative to wild type $(p<0.001)$ (Fig. $3 C, D)$, demonstrating that Mmp2 overexpression is sufficient to block accumulation of Frac. As controls, we scored Frac intensity in embryos with overexpression of a dominant-negative form of $M m p 2, M m p 1$ overexpression, or in embryos mutant for sidestep, a guidance molecule expressed in muscle (Sink et al., 2001). None of these genotypes show significant modifications to Frac levels (Fig. 3E). MMPs often display overlapping substrate specificities; however, these findings indicate that Frac is unlikely to be cleaved by Mmp1. These expression data support our biochemical data and demonstrate that Frac is processed in an Mmp2-dependent manner. Taken with the interaction between Frac and Mmp2 in yeast, we conclude that Frac likely represents an Mmp2 substrate.

\section{frac LOF mutants display ectopic motor axon projections and loosely bundled axons}

Complementary LOF and GOF analyses established that Mmp2 promotes motor axon fasciculation during outgrowth (Fig. $4 J, N$ ) (Miller et al., 2008). Identification of Frac as an Mmp2 substrate prompted us to investigate whether frac was involved in motor axon targeting. Hence, we generated LOF alleles via imprecise excision of a Minos transposon situated in the first intron (Metaxakis et al., 2005) (see Material and Methods). Two alleles were generated with significant deletions of the frac locus (Fig. $1 B$ ). $\mathrm{frac}^{\Delta l}$ is a $1.2 \mathrm{~kb}$ deletion removing part of the second exon. AntiFrac antibodies fail to recognize Frac protein in homozygous mutant embryos, thus confirming it as a protein null (Fig. $4 A, B)$. The second allele, $f r a c^{\Delta 2}$, is a $2.1 \mathrm{~kb}$ deletion removing the first two exons and half of the third exon. However, although the start is deleted, a truncated protein is translated, because $\mathrm{frac}^{\Delta 2}$ is not a protein null (data not shown). Both frac alleles are homozygous viable and display no overt behavioral defects.

We first assayed embryonic development in frac LOF mutants. Because frac is a component of the mesodermal ECM, frac mutants might display gross defects that preclude an interpretation of any axon pathfinding errors. However, our phenotypic analyses argue that this is not the case. First, both frac alleles are homozygous viable, demonstrating that it is not an essential gene. Second, frac mutants have an intact basement membrane as visualized with viking::GFP (Morin et al., 2001; Urbano et al., 2009). Collagen IV, or viking, is expressed by macrophages of the basement membrane, which surround most tissues, including the ventral nerve cord (VNC), brain, and gut (Yasothornsrikul et al., 1997). Both frac alleles display normal localization of viking::GFP, indicating that the basement membrane is not perturbed (Fig. 4C,D and data not shown). Third, appreciable defects in muscle size, number, or epidermal attachment are not observed in frac mutants as visualized with myosin heavy chain antibody (Fig. 4E,F and data not shown) (Kiehart and Feghali, 1986). Thus, we conclude that morphological defects in frac LOF mutants do not interfere with an analysis of motor axon guidance.

We next analyzed motor axon development in frac mutants. In wild type, the intersegmental nerve branch b (ISNb) extends from the CNS bundled with the main branch of the ISN. ISNb axons defasciculate, or separate from, the ISN before the ventrolateral muscle field (VLM). Subsets of ISNb axons innervate the clefts between VLM muscles 7, 6, 13, and 12 (Fig. 4G,K). Embryos mutant for either frac allele have prominent guidance errors. Sixty-four percent of ISNb axons in $\mathrm{frac}^{\Delta 1}$ embryos do not stay tightly bundled and display targeting errors in which individual axons circle back or project to neighboring nerve bundles. These were further classified as defasciculation and/or misprojection errors. Fifty-one percent of ISNb nerves in $\mathrm{rac}^{\Delta 1}$ mutant embryos display hypofasciculated or frayed axons, and 29\% include 

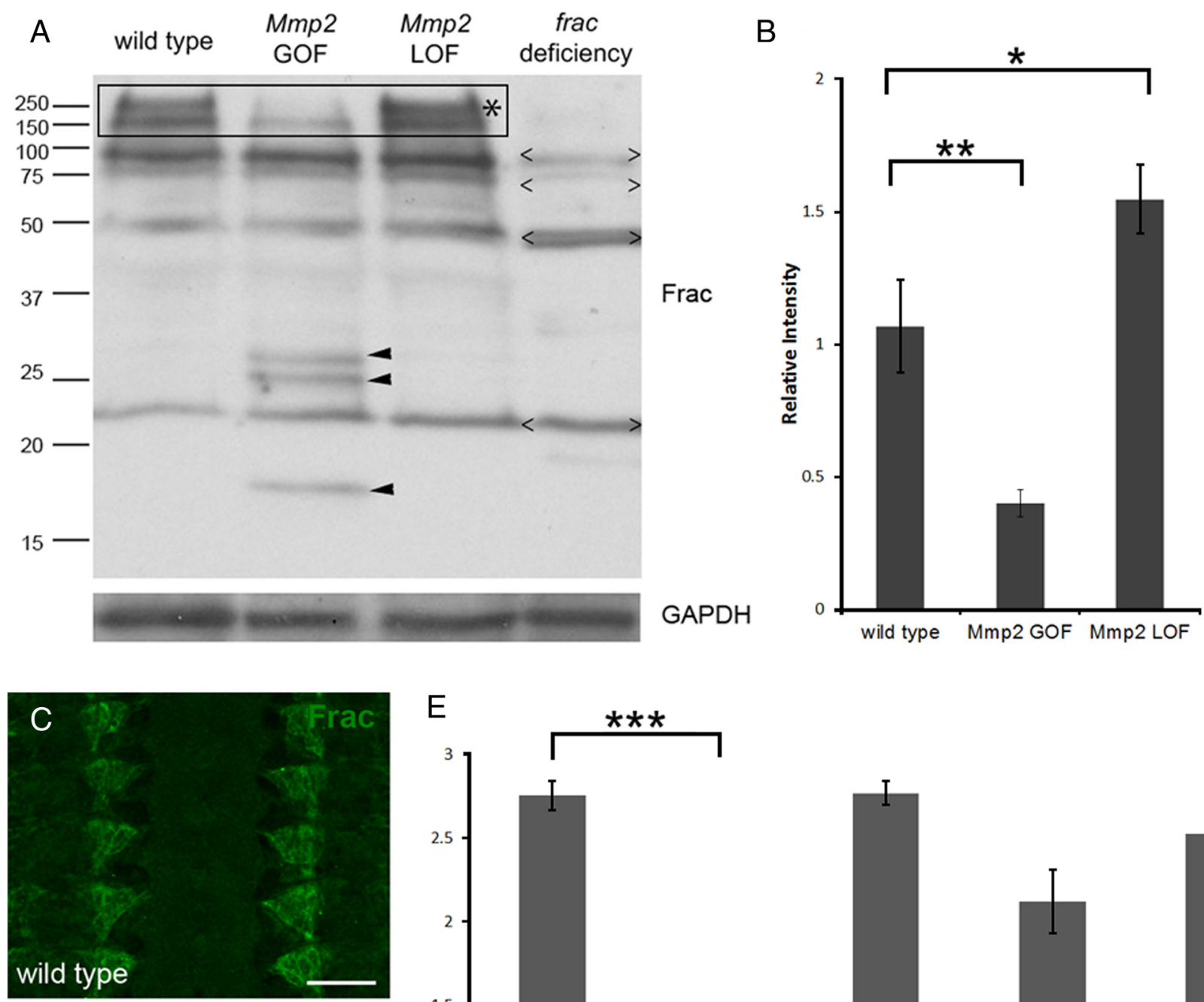

E
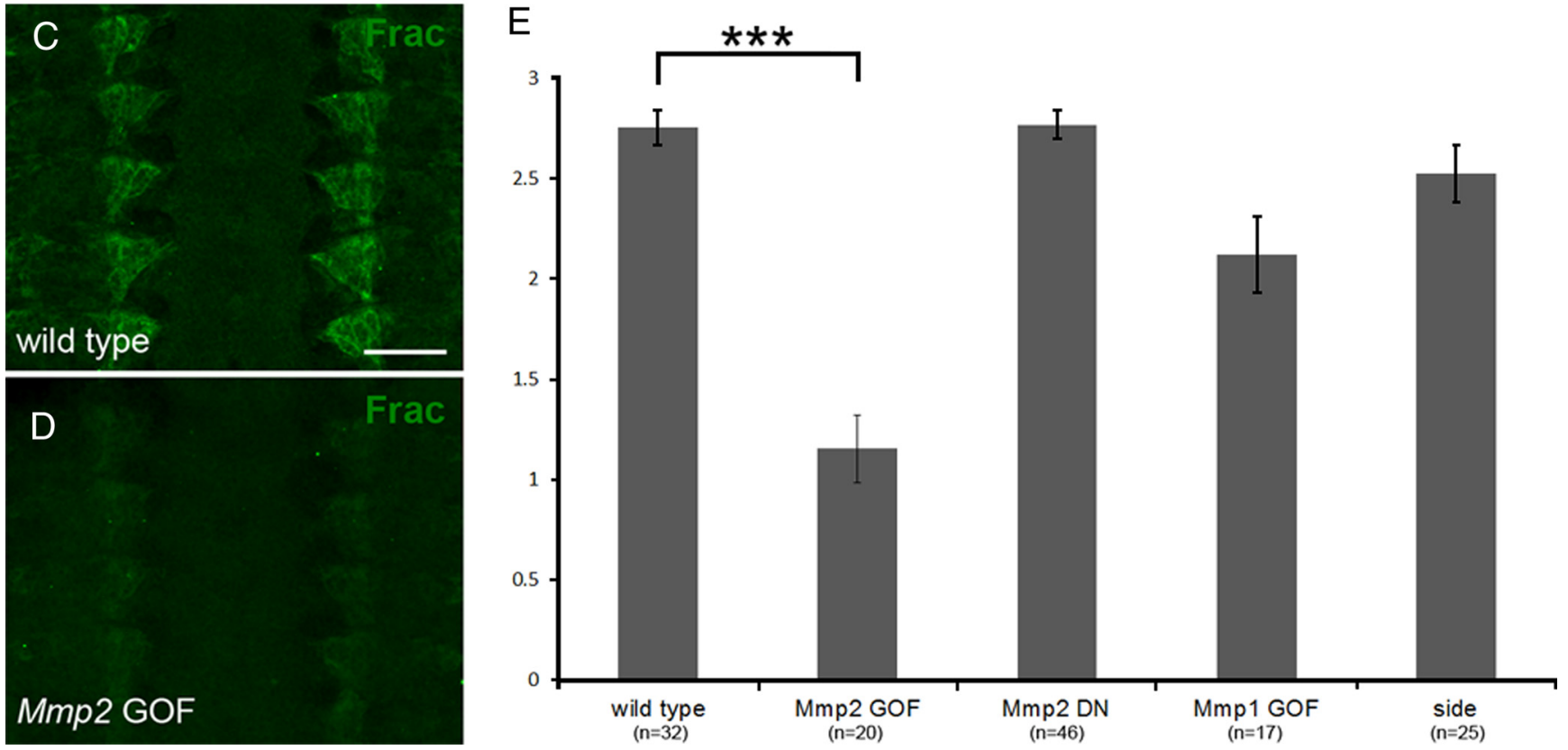

Figure 3. Frac protein processing is Mmp2 dependent. $\boldsymbol{A}$, Western blot with Frac antibody. Bottom shows GAPDH loading control. Full-length Frac is detected in wild-type embryo extracts at 170 and $250 \mathrm{kDa}$. Nonspecific bands are indicated by brackets in the frac deficiency column. In Mmp2 GOF embryos, cleavage products are present at 17, 30, and 33 kDa (arrowheads). $\boldsymbol{B}$, Quantification of the full-length Frac bands (see box in $A$ ) in relation to the density of the $22 \mathrm{kDa}$ background band (significance calculated using ANOVA; $n=9$ blots; ${ }^{*} p<0.05,{ }^{* *} p<0.01$ ). C, D, Stage 14 wild-type $(\boldsymbol{C})$ and tub $>\operatorname{Mmp2}(\boldsymbol{D})$ embryos labeled with Frac antibody. Anterior is up. Scale bar, $50 \mu \mathrm{m}$. $\boldsymbol{E}$, Quantification of Frac protein expression in indicated genotypes. Stage 14 embryos were dissected and scored blindly on a scale of $0-3$ for strength of Frac protein expression. The number of embryos analyzed is indicated below the histogram. Each genotype was individually compared with wild type using the Student's $t$ test $\left.{ }^{* * *} p<0.001\right)$.

axons that make connections with other nerve branches or other ISNb axons (Fig. 4H,L; Table 1). Similarly, in rac $^{\Delta 2}$ mutants, $57 \%$ of hemisegments have aberrant ISNb branches. The same phenotypic classes are present as in $\mathrm{frac}^{\Delta 1}$, with $44 \%$ of ISNb nerves exhibiting ectopic splintering of axons, and 22\% displaying erroneous projections (Fig. 4I, M; Table 1). Both frac alleles are homozygous viable and fertile, so we tested whether maternal contribution of Frac contributes to motor axon behavior. The penetrance of ISNb guidance defects does not increase in the absence of both maternal and zygotic Frac protein ( $p \geq 0.3$ for all) (Table 1), indicating that maternal Frac does not contribute to motor axon pathfinding.

Transposon excisions can result in second site mutations if the transposon excises and briefly integrates elsewhere. Hence, we analyzed $\mathrm{frac}^{\Delta 1} / \mathrm{frac}^{\Delta 2}$ embryos and found that the penetrance remains at $\sim 61 \%$ (Table 1 ). Similarly, we tested each allele over a frac deficiency. Neither $\mathrm{frac}^{\Delta 1} / D f$ nor $\mathrm{rac}^{\Delta 2} / D f$ were statistically different from the respective homozygous mutants $(p \geq 0.7)$ (Table 1). These data indicate that both alleles are genetic nulls, although $\mathrm{frac}^{\Delta 2}$ is not a protein null. The ISNb phenotypes ob- 

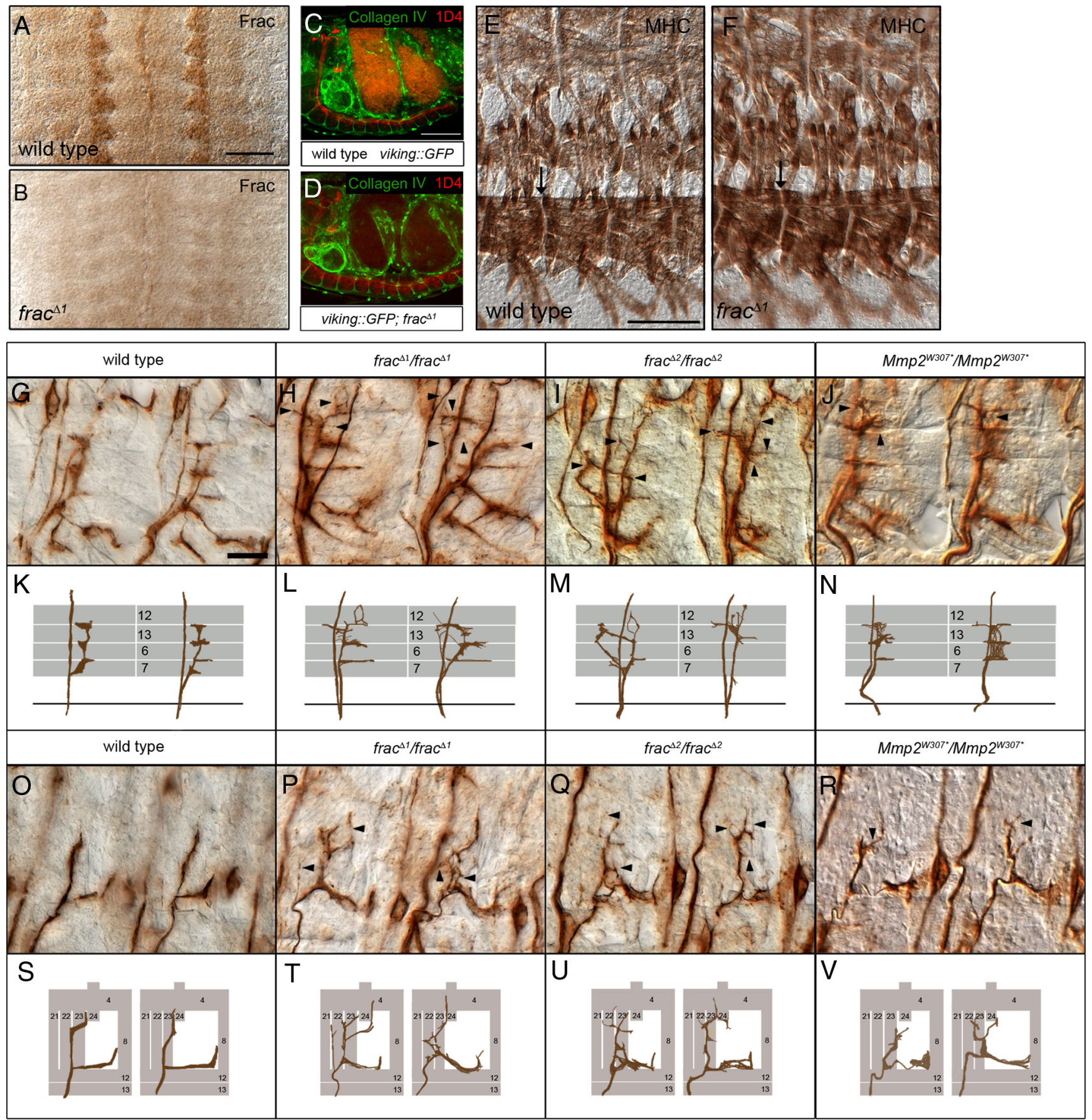

Figure 4. $\quad \boldsymbol{A}, \boldsymbol{B}$, frac LOF mutants display misprojections and defasciculation defects similar to Mmp2 mutants. Stage 14 wild-type $(\boldsymbol{A})$ and frac ${ }^{\Delta 1}(\boldsymbol{B})$ embryos labeled with Frac antibody. $\boldsymbol{C}, \boldsymbol{D}$, Lateral view of stage 16 embryos marked with anti-Fasll, labeling all motor axon projections, and anti-GFP. In addition to the marked genotypes, embryos carry viking::GFP to visualize the cadherin-positive basement membrane. Wild-type $(\boldsymbol{C})$ and frac $^{\Delta{ }^{1}}(\boldsymbol{D})$ homozygous mutant embryos all have Collagen IV (green) expression at the basement membrane surrounding the ventral nerve cord (red) and midgut. $\boldsymbol{E}, \boldsymbol{F}$, MHC antibody was used to visualize the segmentally repeated pattern of muscles in each embryo. In wild-type $(\boldsymbol{E})$ and frac ${ }^{\boldsymbol{1}}(\boldsymbol{F})$ homozygous mutant embryos, muscles are properly formed and attached to the epidermis at muscle attachment sites (arrow). $\mathbf{G}-\boldsymbol{V}$, In each micrograph, two abdominal hemisegments of stage 17 dissected embryos stained with anti-Fasll to label the ISNb $(\mathbf{G}-\boldsymbol{J})$ or $\mathrm{SNa}(\mathbf{O}-\boldsymbol{R})$ motor projections are shown. Below each image are schematics of the branching patterns with the motors axons in brown and the muscles represented

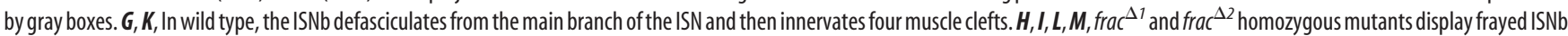
axons and axons that separate at incorrect sites (arrows). $\boldsymbol{I}, \boldsymbol{M}$, In wild type, the SNa extends dorsally to lateral muscles and then bifurcates into posterior and dorsal branches. $\boldsymbol{P}, \mathbf{Q}, \boldsymbol{I}, \boldsymbol{U}, \mathbf{I n}$ frac ${ }^{\Delta l}$ and frac $^{\Delta 2}$ homozygous mutants, axons make projections to aberrant muscle targets and are loosely bundled. $\boldsymbol{J}, \boldsymbol{N}, \boldsymbol{R}, \boldsymbol{V}, \mathbf{M m p 2}{ }^{\mathrm{W3} 07 *}$ mutants display both defasciculation and targeting errors. $\boldsymbol{A}, \boldsymbol{B}$, Anterior is up; $\boldsymbol{E}-\boldsymbol{V}$, anterior is left and dorsal is up. Scale bars: $\boldsymbol{A}-\boldsymbol{D}, 100 \mu \mathrm{m} ; \boldsymbol{E}, \boldsymbol{F}, 50 \mu \mathrm{m} ; \boldsymbol{G}-\boldsymbol{V}, 15 \mu \mathrm{m}$.

served in frac LOF mutants strongly resemble those in Mmp2 LOF mutants and support the hypothesis that Mmp2-dependent regulation of Frac contributes to motor axon targeting.

Mmp2 mutants display defects in the segmental nerve a ( $\mathrm{SNa}$ ) projection in addition to ISNb. To determine whether frac is necessary for SNa projections, we scored SNa guidance in frac mutants. In wild type, the SNa extends dorsally from the CNS to innervate the lateral muscle field. $\mathrm{SNa}$ axons divide into dorsal and posterior branches. The dorsal branch extends between muscles 22 and 23 and divides a second time to innervate muscle 24 . 
Table 1. frac loss-of-function mutants display motor axon defasciculation

\begin{tabular}{|c|c|c|c|c|c|c|}
\hline Genotype & $\begin{array}{l}\text { Total } \\
\text { defasciculated ISNb }{ }^{a}(n)\end{array}$ & Misprojection & Hypofasciculation & $\begin{array}{l}\text { Total } \\
\text { defasciculated SNa }{ }^{a}(n)\end{array}$ & Misprojection & Hypofasciculation \\
\hline $\operatorname{Mi}\{\mathrm{ET} 1\} C G 7526^{\mathrm{MB} 05690}$ & $13 \%(160)$ & $3 \%$ & $11 \%$ & $11 \%(152)$ & $1 \%$ & $11 \%$ \\
\hline$M m p 2^{W 307^{*}}$ & $81 \%(133)$ & $23 \%$ & $79 \%$ & $42 \%(127)$ & $5 \%$ & $37 \%$ \\
\hline frac $^{\Delta 1}$ zygotic & $64 \%(119)$ & $29 \%$ & $51 \%$ & $49 \%(119)$ & $9 \%$ & $46 \%$ \\
\hline frac $^{\Delta 1}$ maternal/zygotic & $60 \%(250)$ & $22 \%$ & $52 \%$ & $43 \%(249)$ & $4 \%$ & $41 \%$ \\
\hline $\mathrm{frac}^{\Delta 1} / \mathrm{Df}(3 \mathrm{~L}) \mathrm{pbl}-\mathrm{X} 1$ & $68 \%(102)$ & $25 \%$ & $50 \%$ & $36 \%(101)$ & $3 \%$ & $34 \%$ \\
\hline frac $^{\Delta 2}$ zygotic & $57 \%(117)$ & $22 \%$ & $44 \%$ & $45 \%(117)$ & $3 \%$ & $43 \%$ \\
\hline frac $^{\Delta 2}$ maternal/zygotic & $58 \%(183)$ & $25 \%$ & $44 \%$ & $40 \%(180)$ & $4 \%$ & $47 \%$ \\
\hline $\operatorname{frac}^{\Delta 2} / \mathrm{Df}(3 \mathrm{~L}) \mathrm{pbl}-\mathrm{X} 1$ & $57 \%(190)$ & $25 \%$ & $47 \%$ & $35 \%(175)$ & $2 \%$ & $35 \%$ \\
\hline $\operatorname{frac}^{\Delta 1} /$ frac $^{\Delta 2}$ & $61 \%(100)$ & $24 \%$ & $52 \%$ & $39 \%(100)$ & $5 \%$ & $34 \%$ \\
\hline $\operatorname{Sema}^{P 1} a^{P 1}+;$ frac $^{\Delta 1} /$ frac $^{\Delta 1}$ & $18 \%(177)$ & $7 \%$ & $11 \%$ & $9 \%(155)$ & $1 \%$ & $8 \%$ \\
\hline Sema1a ${ }^{\mathrm{Df}(2 \mathrm{~L}) \mathrm{N} 22-5} /+;$ frac $^{\Delta 1} / \mathrm{frac}^{\Delta 1}$ & $30 \%(92)$ & $4 \%$ & $24 \%$ & $16 \%(90)$ & $1 \%$ & $14 \%$ \\
\hline beat1a ${ }^{163 c} /+$; frac ${ }^{\Delta 1} /$ frac $^{\Delta 1}$ & $30 \%(159)$ & $4 \%$ & $26 \%$ & $18 \%(148)$ & $1 \%$ & $18 \%$ \\
\hline
\end{tabular}

$n$, The number of hemisegments scored for each genotype.

${ }^{a}$ Hemisegments may have defects in both misprojection and hypofasciculation, yielding a total defasciculation that is not the sum of the two separate categories.

The posterior branch extends to innervate muscle 8 (Fig. 4O,S). In control embryos, $11 \%$ of hemisegments display hypofasciculation or misprojection defects in the $\mathrm{SNa}$ (Table 1). In contrast, $49 \%$ of hemisegments in $\mathrm{frac}^{\Delta 1}$ embryos and $45 \%$ in $\mathrm{frac}^{\Delta 2} \mathrm{em}-$ bryos contain axons that separate inappropriately and project ectopically ( $p<0.001$ for both compared with wild type) (Fig. $4 P, Q, T, U$; Table 1$)$. The majority of defects are hypofasciculation errors in which axons inappropriately branch away from the $\mathrm{SNa}$, occurring in $\mathrm{frac}^{\Delta 1}$ and $43 \%$ in $\mathrm{frac}^{\Delta 2}$ (Table 1). Most SNa nerves fray near choice points, in which nerve bundles divide and axons follow different pathways to innervate their specific targets. The SNa hypofasciculation phenotypes observed in frac mutants resemble those in $M m p 2$ mutants and suggest that Mmp2 regulates Frac processing to modulate motor axon fasciculation in multiple motor tracks (Fig. $4 R, V$; Table 1). A priori, Mmp2dependent cleavage could lead to either substrate activation or inactivation. Because $M m p 2$ and frac mutants both display loosely bundled axons with ectopic projections, we propose that Mmp2-dependent cleavage activates Frac to promote motor axon bundling.

The pathfinding phenotype in frac LOF mutants argues that frac promotes motor axon fasciculation. Appropriate levels of motor axon bundling require a balance between interaxonal adhesion and attraction between motor axons and their targets (Winberg et al., 1998; Yu et al., 1998, 2000). The Sema1a/PlexA repulsive signaling pathway plays a central role in this process. LOF mutants in the pathway display increased motor axon bundling and fail to properly innervate their targets (Yu et al., 1998), a phenotype roughly opposite to that displayed by frac LOF mutants. We asked whether frac displays genetic interactions with Semala to obtain additional evidence that frac enhances fasciculation. Sema la dominantly suppresses the ISNb pathfinding phenotypes from $64 \%$ in frac homozygotes to $18 \%$ in frac mutants that are also heterozygous for Semala $a^{P 1}(p<0.001)$ (Table 1). This interaction is not allele specific because the frac LOF phenotypes are suppressed by two independent Semala alleles (Table $1)$. To test whether this genetic interaction reflects a specific relationship between frac and Semala, we asked whether frac mutants also display interactions with beat 1 , another secreted guidance cue. Motor axons in homozygous beat1a embryos display increased fasciculation (Fambrough and Goodman, 1996). We find that beat 1 a dominantly suppresses frac defasciculation defects (Table 1), indicating that frac activity regulates the degree of attraction between motor axons. The genetic interactions between frac, Semala, and beat 1 a further argue that the pathfinding defects observed in frac mutants reflect a specific role for frac in guidance and are not the secondary consequence of morphological defects.

\section{Frac and Mmp2 interact genetically}

These genetic analyses establish that frac is required for axon targeting, and the biochemical data argue that Frac is cleaved in an Mmp2-dependent manner. To test whether Mmp2 and Frac interact in vivo to control axon guidance, we undertook genetic interaction experiments. Because genes acting in common genetic pathways often exhibit dose-dependent genetic interactions, we asked whether $M m p 2$ and frac display dominant genetic interactions. Embryos doubly heterozygous for $M m p 2$ and frac exhibit elevated levels of ISNb and SNa guidance defects (Fig. 5B; Table 2). For example, the incidence of ISNb misprojection rises from 1 and $2 \%$ in frac and $M m p 2$ heterozygotes, respectively, to $18 \%$ in Mmp2; frac double heterozygotes ( $p<0.001$ for both). These interaction data support the hypothesis that Mmp2 and Frac cooperate to regulate motor axon targeting, but they do not address the question of their relative order of action. To ask whether $M m p 2$ and frac act in a linear genetic pathway, we asked whether the increased motor axon fasciculation in Mmp2 overexpression embryos depends on frac activity. Pan-glial Mmp2 overexpression strongly increases ISNb and $\mathrm{SNa}$ fasciculation (Fig. 5E, G) (Miller et al., 2008), a phenotype distinct from the decreased fasciculation in frac LOF embryos. If Frac is an Mmp2 substrate, the Mmp2 overexpression phenotype is predicted to require Frac, and the frac LOF phenotype would be epistatic to the Mmp2 overexpression phenotype. To test this model, we overexpressed Mmp2 in a frac homozygous mutant background and found that the excess fasciculation in Mmp2 overexpressing embryos is completely suppressed (63\% ISNb hyperfasciculation in repo $>M m p 2$ to $3 \%$ ISNb hyperfasciculation in $\mathrm{frac}^{\Delta 1}$, repo $>M m p 2 ; p<0.001$ ) (Fig. 5F,H; Table 2). Furthermore, these embryos display diminished motor axon bundling similar to that observed in frac homozygotes (Figs. $4 H, L, 5 F, H$; Table 2). Hence, frac is genetically downstream of $M m p 2$, consistent with our biochemical data identifying Frac as an Mmp2 substrate.

These studies argue that Mmp2 cleaves Frac to promote motor axon fasciculation and raise the question of the identity of the Mmp2-generated pro-fasciculation signal. A matrix molecule such as Frac may influence signaling by a variety of means (Hynes, 2009). We can envision three likely relationships between Frac and the guidance signal. First, it is possible that fulllength Frac sequesters a soluble guidance cue. In this case, Mmp2 


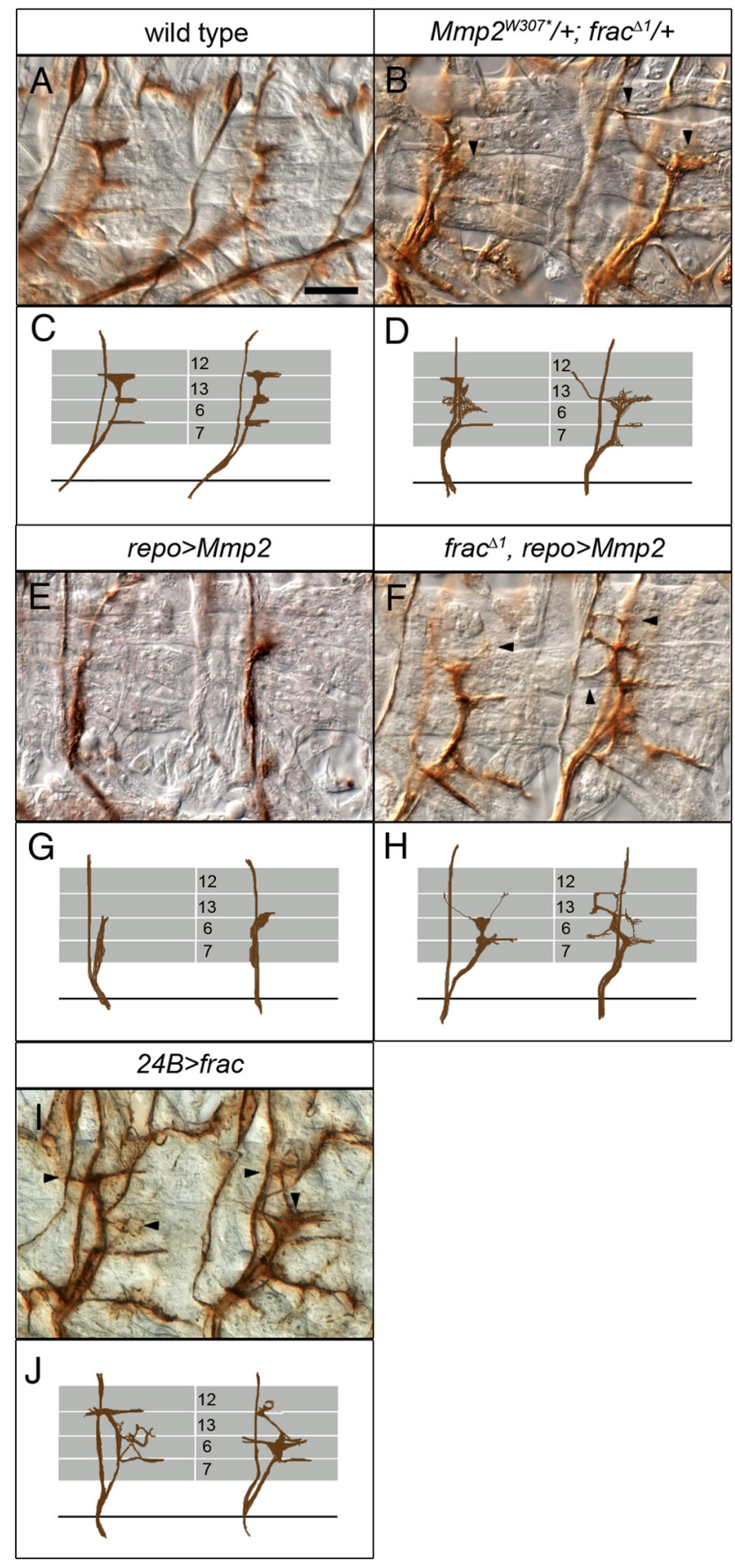

Figure 5. frac is genetically downstream of Mmp2 in axon guidance. Two abdominal hemisegments of stage 17 embryos labeled with anti-Fasll are shown with a schematic of the phenotype shown below. $\boldsymbol{A}, \boldsymbol{C}$, Wild-type embryos have tightly bundled axonal projections. $\boldsymbol{B}$, D, In Mmp2 $2^{\text {W307*' }+ \text {; frac }}{ }^{\Delta 1} /+$ embryos, the ISNb displays defasciculation defects. $\boldsymbol{E}, \mathbf{G}$, repo $>M m p 2$ embryos exhibit ISNb axons that are hyperfasciculated and fail to reach their targets. $\boldsymbol{F}, \boldsymbol{H}$, frac $^{\Delta 1}$, repo $>M m p 2$ embryos have axons that misproject and are defasciculated. $I, J, \ln 24 B>$ frac embryos, the ISNb displays extra projections and misrouted axons (arrows). Anterior is left and dorsal is up in all. Scale bar, $15 \mu \mathrm{m}$.

cleavage liberates the signaling molecule from full-length Frac. frac LOF would then be characterized by elevated guidance factor signaling (no Frac to sequester cues), and Mmp2 LOF would be characterized by decreased guidance factor signaling (increased Frac to sequester cues). The finding that the guidance phenotypes displayed by Mmp2 LOF and frac LOF mutants strongly resemble each other argues against the model that Frac is simply a sink for an active guidance factor. Alternatively, the Frac fragment may have intrinsic signaling activity and act as a repulsive mesodermbased cue to keep motor axons tightly bundled. As a third possibility, the Frac cleavage fragment may be a cofactor in a signaling complex with a guidance cue.

To distinguish between the second and third signaling mechanisms, we overexpressed Frac throughout the mesoderm. If the Frac cleavage fragment has intrinsic signaling activity, then we expect frac overexpression to lead to elevated levels of axon bundling because the axons are exposed to more pro-fasciculation signal. However, if the Frac cleavage fragment represents only part of a signaling complex, then frac overexpression would not lead to hyperfasciculation because the Frac fragment is insufficient to signal alone. We find that frac overexpression does not promote hyperfasciculation; in fact, the motor axons in $24 \mathrm{~B}>\mathrm{frac}$ embryos are poorly fasciculated and project ectopically (Fig. 5I, J; Table 2). This phenotype is observed with independent UAS-frac lines (Table 2), indicating that it is not a secondary effect of insertion site. The hypofasciculation observed in these embryos suggests that frac overexpression overwhelms normal regulation by $\mathrm{Mmp} 2$ and attenuates an active Frac fragment/signaling cue complex. This phenotype demonstrates that Frac is not sufficient to promote fasciculation and hints at the existence of a signaling cue associated with the Frac cleavage fragment.

\section{Frac regulates a LIMK-dependent BMP signaling pathway}

The motor axon phenotypes associated with frac overexpression indicate that frac is not sufficient to promote fasciculation and suggest the presence of a frac-regulated pathfinding cue. Studies in vertebrate systems provide ample evidence that the related Fibrillin family controls the bioavailability of growth factors, most notably TGF $\beta /$ BMP family members (Neptune et al., 2003; Charbonneau et al., 2004; Chaudhry et al., 2007; Sengle et al., 2008). To test whether frac modulates BMP signaling, we investigated genetic interactions between frac and the BMP pathway. With the exception of the activin-like protein Dawdle (Parker et al., 2006; Serpe and O'Connor, 2006), there is a lack of published evidence for BMP involvement in motor axon guidance. However, many of the BMP pathway mutants have pleiotropic embryonic defects, complicating LOF analysis (Khalsa et al., 1998; Wharton et al., 1999).

We first asked whether an activated form of the type I receptor saxophone (sax) modulates the frac LOF phenotype (Haerry et al., 1998). Pan-neuronal overexpression of sax ${ }^{a c t}$ does not yield appreciable motor axon phenotypes (Fig. $6 B, E$; Table 3 ), yet sax ${ }^{\text {act }}$ suppresses the frac LOF phenotype, reducing the incidence of defasciculation from 64 to 30\% $(p<0.001)$ (Fig. 6A, C,D,F; Table 3 ), suggesting that BMP signaling is compromised in a frac LOF mutant background. Similar to frac LOF, frac overexpression embryos are characterized by reduced levels of ISNb fasciculation. In this case, we argue that Frac overexpression may overcome Mmp2-dependent regulation and effectively sequester a guidance cue. If this cue is a BMP, then expression of sax $x^{\text {act }}$ in a frac overexpression background is predicted to suppress ISNb defasciculation. Indeed, motor axon misprojections and hypofasciculation in frac overexpression is partially suppressed by cooverexpression of sax ${ }^{a c t}$ (55 to $27 \% ; p<0.001$ ) (Table 3). Suppression of the frac pathfinding phenotypes via neuronal activation of the BMP pathway argues that frac activity normally promotes BMP pathway activation in motoneurons. In line with this view, mesodermal overexpression of the BMP ligand $g b b$ suppresses the phenotype observed with frac overexpression (48 to $28 \%$; $p<0.001$ ) (Table 3) (Khalsa et al., 1998), providing 
Table 2. frac and Mmp2 genetically interact

\begin{tabular}{|c|c|c|c|c|c|c|c|c|}
\hline Genotype & $\begin{array}{l}\text { Total } \\
\text { defasciculated ISNb }{ }^{a}(n)\end{array}$ & Misprojection & Hypofasciculation & Hyperfasciculation & $\begin{array}{l}\text { Total } \\
\text { defasciculated SNa }{ }^{a}(n)\end{array}$ & Misprojection & Hypofasciculation & Hyperfasciculation \\
\hline Mi\{ET1\}CG7526 MB05690 & $13 \%(160)$ & $3 \%$ & $11 \%$ & $0 \%$ & $11 \%(152)$ & $1 \%$ & $11 \%$ & $0 \%$ \\
\hline$M m p 2^{W 307^{*}}$ & $81 \%(133)$ & $23 \%$ & $79 \%$ & $0 \%$ & $42 \%(127)$ & $5 \%$ & $37 \%$ & $0 \%$ \\
\hline frac $^{\Delta 1}$ zygotic & $64 \%(119)$ & $29 \%$ & $51 \%$ & $0 \%$ & $49 \%(119)$ & $9 \%$ & $46 \%$ & $0 \%$ \\
\hline$M m p 2^{W 307^{*}} /+$ & $15 \%(140)$ & $2 \%$ & $13 \%$ & $0 \%$ & $5 \%(126)$ & $0 \%$ & $5 \%$ & $0 \%$ \\
\hline $\operatorname{frac}^{\Delta 1} /+$ & $21 \%(160)$ & $1 \%$ & $19 \%$ & $0 \%$ & $6 \%(136)$ & $0 \%$ & $6 \%$ & $0 \%$ \\
\hline $\operatorname{Mmp} 2^{W 307^{*}} /+; \operatorname{frac}^{\Delta 1} /+$ & $46 \%(128)$ & $18 \%$ & $38 \%$ & $0 \%$ & $16 \%(122)$ & $1 \%$ & $16 \%$ & $0 \%$ \\
\hline repo $>M m p 2^{b}$ & $3 \%(120)$ & $0 \%$ & $3 \%$ & $63 \%$ & $9 \%(109)$ & $0 \%$ & $9 \%$ & $61 \%$ \\
\hline frac $^{\Delta 1}$, repo $>M m p 2$ & $47 \%(234)$ & $3 \%$ & $45 \%$ & $3 \%$ & $21 \%(215)$ & $1 \%$ & $20 \%$ & $1 \%$ \\
\hline $24 B>$ frac \#3 & $63 \%(120)$ & $16 \%$ & $53 \%$ & $0 \%$ & $39 \%(115)$ & $6 \%$ & $33 \%$ & $0 \%$ \\
\hline $24 B>$ frac \#7 & $57 \%(221)$ & $17 \%$ & $48 \%$ & $0 \%$ & $30 \%(214)$ & $3 \%$ & $28 \%$ & $0 \%$ \\
\hline
\end{tabular}

$n$, The number of hemisegments scored for each genotype.

${ }^{a}$ Hemisegments may have defects in both misprojection and hypofasciculation, yielding a total defasciculation that is not the sum of the two separate categories.

${ }^{b}$ Data reported by Miller et al. (2008).

additional genetic evidence that frac overexpression attenuates signaling via a Frac/BMP complex. However, the incomplete suppression in these assays suggests that frac regulates additional guidance cues.

Classical BMP signaling results in phosphorylation of the transcription factor Mad, which translocates to the nucleus and activates gene transcription (Marqués et al., 2002). BMP signaling is required for neuromuscular junction growth in Drosophila, and pMad has been shown to accumulate in motoneurons. Motoneuronal pMad is apparent during embryogenesis, raising the possibility that this pathway plays a role in targeting. To test whether Mad-mediated BMP signaling is regulated by frac, we examined pMad expression in frac LOF and GOF embryos. We do not detect an obvious difference in the number of pMadpositive neurons or in pMad intensity in either LOF or GOF frac mutants (Fig. 6G-I). These results suggest that frac regulates a noncanonical BMP signaling pathway. LIMK1 is downstream of the BMP type 2 receptor Wishful thinking at the neuromuscular junction, in which it is essential for synaptic stability (Eaton and Davis, 2005). Thus, LIMK1 represented an attractive candidate to be involved in a Frac/BMP-dependent, pMad-independent pathway in motor axons. Indeed, LIMK1 homozygous mutants display defasciculation defects qualitatively similar to that of frac LOF mutants (Fig. 6J, M; Table 3). In support of a neuronal function for LIMK1 in guidance, neuronal-specific expression of LIMK1 RNAi constructs also drives ISNb defasciculation (Fig. $6 \mathrm{~K}, \mathrm{~N}$; Table 3 ). To test whether frac and $L I M K 1$ are likely to act in a common genetic pathway, we quantified defasciculation in LIMK1; frac double homozygotes. Consistent with this hypothesis that LIMK1 is downstream of a Frac/BMP signaling complex, the frequency of defasciculation in LIMK1; frac double mutants is not significantly elevated over that observed in frac homozygotes (64 to $58 \%$; $p>0.3$ ) (Fig. $6 \mathrm{~L}, \mathrm{O}$; Table 3). Moreover, embryos doubly heterozygous for LIMK1 and frac display an increase in misprojections relative to either heterozygote alone ( 9 to $1 \%$; $p<$ 0.05) (Table 3). Thus, we conclude that Frac acts via an LIMK1dependent noncanonical BMP pathway to promote proper motor axon pathfinding.

\section{Discussion}

Signaling downstream of axon guidance receptors is tuned precisely to generate proper connectivity. Elucidating the mechanisms allowing for stereotyped local activation of these pathways remains a major challenge. The ECM is perfectly situated to provide extracellular cues that could impinge on targeting and provide the spatiotemporal signals to regulate events such as fasciculation, branching, or synaptogenesis. However, the interactions between matrix molecules and guidance receptors remain poorly defined. Here we demonstrate that the novel ECM molecule Frac regulates motor axon guidance. Furthermore, Frac itself is regulated via proteolysis, adding another layer of regulation to ensure local activation of guidance receptor pathways.

Frac, an ECM molecule structurally related to vertebrate Fibrillins and Fibulins, was identified in a yeast interaction screen for Mmp2-binding proteins. Frac is expressed in the embryonic mesoderm concurrent with axon pathfinding and directly adjacent to Mmp2-expressing exit glia. We further show that Frac processing in embryos is Mmp2 dependent. frac LOF alleles display marked defects in axon pathfinding, which are tantamount to those displayed by Mmp2 LOF mutants, providing evidence that Mmp2 cleaves and activates Frac (Fig. 7). We undertook genetic interaction analyses to elucidate the mechanism of Mmp2-Frac signaling. These studies argue that (1) the Frac fragment generated by Mmp2 does not have inherent signaling activity, and (2) frac contributes to the activation of a noncanonical BMP signaling pathway in motoneurons. To our knowledge, these data are the first to demonstrate that proteolysis of an ECM molecule is involved in regulating the distribution or activation of a signaling cue during axon guidance.

Motor axons in Drosophila selectively defasciculate at guidance choice points as they follow individual routes to their synaptic targets. Repulsive signaling driven by the Semaphorin1a-PlexinA pathway acts in motor axons to promote interaxonal repulsion essential for axon separation (Yu et al., 1998; Terman and Kolodkin, 2004). However, the mechanism by which defasciculation is normally confined to axon choice points is unknown. We have presented evidence that an Mmp2-Frac pathway is necessary to limit axon defasciculation. Together, our data argue that a Frac cleavage product acts in the mesodermal ECM to signal axons to remain bundled. Because Mmp2 regulates Frac processing, signal activation may be precisely modulated both spatially and temporally. We hypothesize that the Mmp2-Frac pathway provides a cue to axons to remain bundled during outgrowth and overcomes the interaxonal repulsion driven by the Sema1a-PlexA pathway. This antagonistic relationship receives strong support from the finding that both Mmp2 and Frac are dominantly suppressed by Semala mutations (Table 1) (Miller et al., 2008). We speculate that $M m p 2$ is inactive at choice points, allowing the Sema1a-PlexA pathway to promote motor axon separation at these locations. 
Metalloproteinase function in the developing nervous system

Among the metalloproteinases, the ADAM (a disintegrin and metalloproteinase domain) family has been most intimately linked to the regulation of axon targeting to date. In particular, the transmembrane protein ADAM10/Kuzbanian (Kuz) regulates the ectodomain shedding of a number of neural substrates, including the Roundabout receptor, Notch, and GPIlinked Ephrin A2 (Hattori et al., 2000; Mumm and Kopan, 2000; Coleman et al., 2010). Given the central role that proteolysis is likely to play in sculpting nervous system connectivity and function, why are there few examples of MMP activity in this process? Analysis of the MMP family in neuronal development in vertebrates has likely been primarily obscured by the functional redundancy among the 24 MMP family members. Broad-spectrum MMP inhibitors and compound mouse mutants are beginning to resolve their functions. For example, Hehr et al. (2005) used MMP inhibitors in an analysis of retinal ganglion cell guidance in Xenopus and found evidence for MMP function at two distinct choice points. There is also emerging evidence that MMP-9 elicits stable modifications of spine structure in long-term potentiation via ECM remodeling (Bozdagi et al., 2007; Wang et al., 2008b), arguing that synaptic plasticity requires MMPs.

In Drosophila, Mmp1 is secreted whereas Mmp2 is anchored to the membrane via a GPI link. The membrane association of Mmp2 may position it well to regulate defined aspects of neuronal development. In addition to the work presented here, $M m p 2$ is required for dendritic reshaping of sensory neurons in the adult (Yasunaga et al., 2010). These authors found that a pulse of Mmp2 expression in epithelial cells directly apposed to a class of adult sensory neurons is coincident with the dendritic remodeling. Furthermore, dendritic reshaping is blocked in $\mathrm{Mmp} 2$ mutants, and clonal analysis indicates that $M m p 2$ acts locally in dendritic morphogenesis and reshapes only those dendrites in direct contact with Mmp2-positive epithelia. Hence, this work provides a second example of a local function of $\mathrm{Mmp2}$ defining neuronal morphology in development. Given the specialized microenvironments likely present in the neuronal ECM, the spatiotemporal control of proteolysis provided by a membrane-associated MMP is predicted to be of fundamental importance.

\section{An Mmp2-Frac signaling pathway}

This study has uncovered an Mmp2-Frac signaling module that promotes proper motor axon targeting. Motor axons have a precisely modulated attraction for their mesodermal ECM substrate.

M
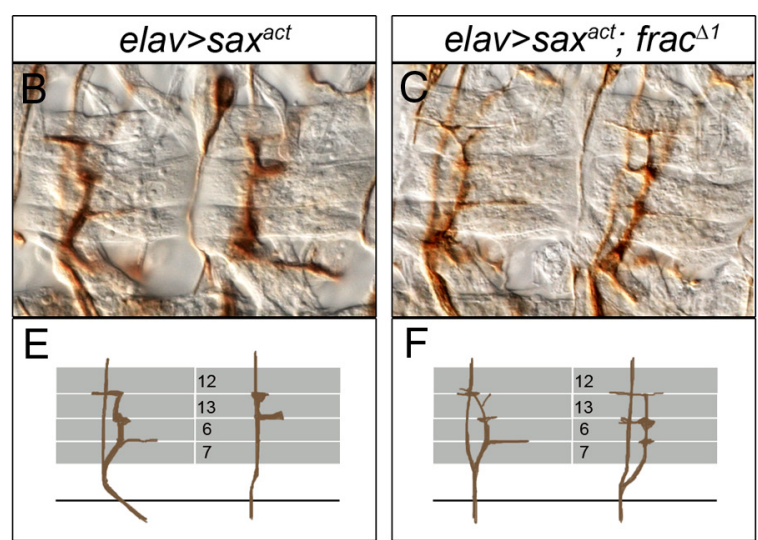

F
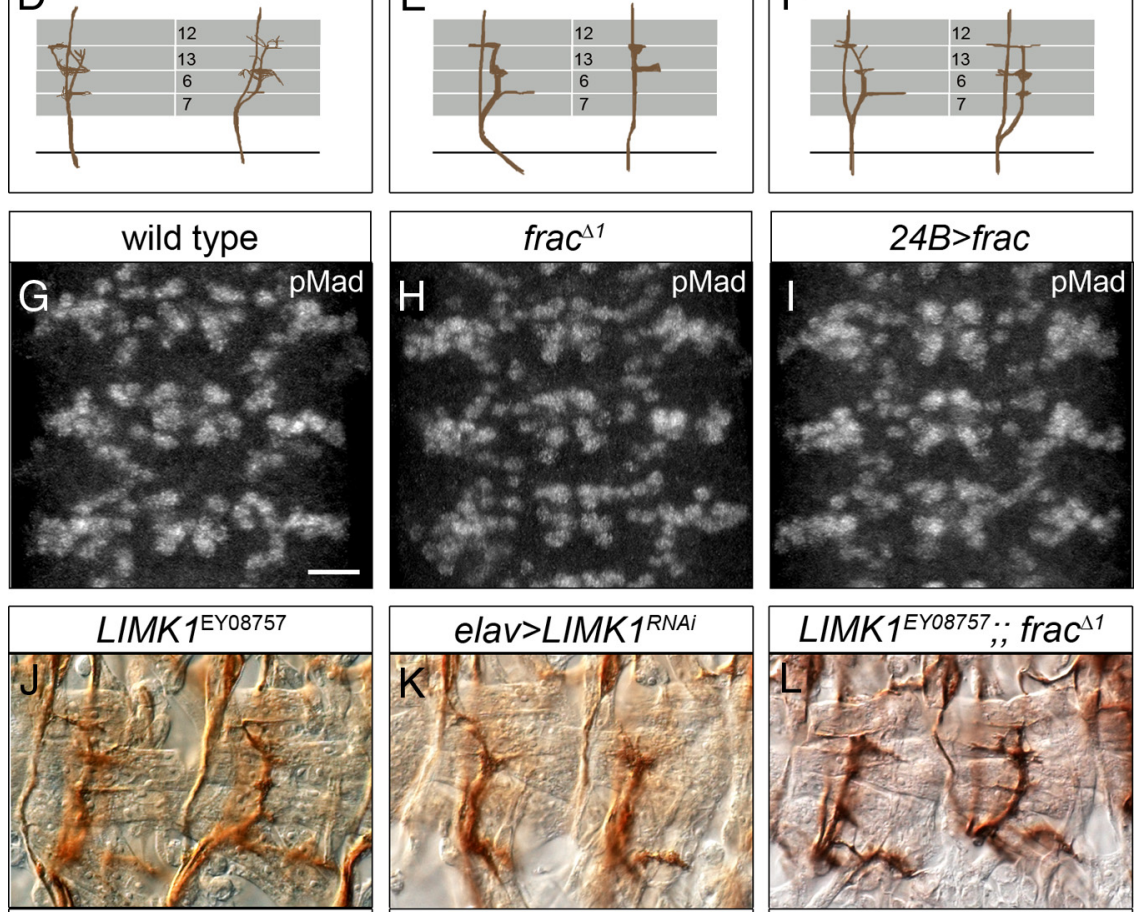

$\mathrm{N}$

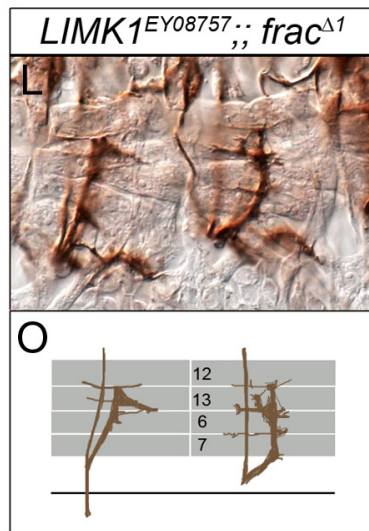

Figure 6. Frac signals downstream through an LIMK-dependent BMP signaling mechanism. $\boldsymbol{A}-\mathbf{C}, \mathbf{J}-\mathbf{L}$, Two abdominal hemisegments of stage 17 embryos stained with anti-Fasll to mark motor axon projections. $\boldsymbol{A}, \boldsymbol{D}$, frac ${ }^{\Delta 1}$ mutant embryos display hypofasciculation and misprojection errors. $\boldsymbol{B}, \boldsymbol{E}$, In embryos expressing constitutively active Sax, the ISNb innervates its proper targets. $\boldsymbol{C}, \boldsymbol{F}$, Homozygous frac ${ }^{\Delta 1}$ mutant embryos expressing elav $>$ sax ${ }^{a c t}$ reach their targets with some defasciculation errors. Stage 16 wild-type $(\boldsymbol{G})$, frac ${ }^{\Delta 1}$ homozygous mutant $(\boldsymbol{H})$, and $24 B>$ frac $(\boldsymbol{I})$ embryonic nerve cords labeled with pMad antibody. $\boldsymbol{J}$ $M$, LIMK1 ${ }^{\mathrm{EY} 08757}$ homozygous mutants exhibit defasciculation errors. $\boldsymbol{K}, \boldsymbol{N}$, In embryos with neuronal RNAi knockdown of LIMK1 with concurrent neuronal misexpression of UAS-Dicer2, the ISNb displays targeting errors. L, O, The ISNb of LIMK1 ${ }^{\text {EY08757; }}$;rac ${ }^{\Delta 1}$ double mutant embryos is defasciculated and has ectopic projections. $\mathbf{A - F , J - 0 , ~ A n t e r i o r ~ i s ~ l e f t ~ a n d ~ d o r s a l ~ i s ~ u p . ~ S c a l e ~ b a r : ~} 15 \mu \mathrm{m}$. G-I, Anterior is up. Scale bars, $20 \mu \mathrm{m}$.
They must be sufficiently attracted to the mesodermal ECM to leave the ventral nerve cord and initiate migration on it, yet they need to limit exploration on this substrate and maintain strong interaxonal adhesion. Our data argue that an Mmp2-dependent Frac cleavage fragment keeps motor axons on track. Because Mmp2 is expressed by motor-axon-associated exit glia, this processing event is positioned only in the vicinity of extending axons (Fig. 7). In general terms, the ECM is positioned to regulate the distribution, activation, and presentation of growth factors, raising a number of possibilities for the role of Frac in axon guidance.

A relatively straightforward model based on the role of the related Fibrillin family in vertebrates is that Frac acts as a reservoir or sink for a guidance cue, which is released to signal via Mmp2dependent cleavage. Vertebrate Fibrillins play major functional 
Table 3. frac functions upstream of a BMP signaling pathway

\begin{tabular}{|c|c|c|c|c|c|c|}
\hline Genotype & $\begin{array}{l}\text { Total } \\
\text { defasciculated ISNb }{ }^{a}(n)\end{array}$ & Misprojection & Hypofasciculation & $\begin{array}{l}\text { Total } \\
\text { defasciculated SNa }{ }^{a}(n)\end{array}$ & Misprojection & Hypofasciculation \\
\hline $\operatorname{Mi}\{E T 1\} C G 7526^{\mathrm{MB} 05690}$ & $13 \%(160)$ & $3 \%$ & $11 \%$ & $11 \%(152)$ & $1 \%$ & $11 \%$ \\
\hline frac $^{\Delta 1}$ zygotic & $64 \%(119)$ & $29 \%$ & $51 \%$ & $49 \%(119)$ & $9 \%$ & $46 \%$ \\
\hline elav $>$ sax ${ }^{a c t}$ & $17 \%(129)$ & $3 \%$ & $14 \%$ & $10 \%(128)$ & $1 \%$ & $9 \%$ \\
\hline elav $>\operatorname{sax}^{a c t}$, frac $^{\Delta 1}$ & $30 \%(147)$ & $4 \%$ & $29 \%$ & $28 \%(137)$ & $0 \%$ & $28 \%$ \\
\hline elav, 24B $>$ sax $x^{a c t}$ & $26 \%(135)$ & $4 \%$ & $24 \%$ & $11 \%(121)$ & $3 \%$ & $8 \%$ \\
\hline elav, 24B >frac \#7 & $55 \%(132)$ & $9 \%$ & $49 \%$ & $28 \%(128)$ & $6 \%$ & $30 \%$ \\
\hline elav, $24 B>$ sax ${ }^{a c t}$, frac \#7 & $27 \%(107)$ & $4 \%$ & $25 \%$ & $14 \%(100)$ & $2 \%$ & $14 \%$ \\
\hline $24 \mathrm{~B}>g b b$ & $20 \%(174)$ & $4 \%$ & $16 \%$ & $2 \%(173)$ & $0 \%$ & $2 \%$ \\
\hline $24 \mathrm{~B}>g b b$, frac \#7 & $28 \%(199)$ & $7 \%$ & $23 \%$ & $9 \%(193)$ & $1 \%$ & $9 \%$ \\
\hline $24 \mathrm{~B}>L Z$, frac \#7 & $48 \%(174)$ & $2 \%$ & $48 \%$ & $15 \%(147)$ & $1 \%$ & $14 \%$ \\
\hline$L I M K 1^{E Y 08757}$ & $51 \%(237)$ & $20 \%$ & $46 \%$ & $29 \%(237)$ & $2 \%$ & $28 \%$ \\
\hline$L I M K 1^{D f(1) H F 368}$ & $47 \%(126)$ & $11 \%$ & $37 \%$ & $29 \%(120)$ & $5 \%$ & $26 \%$ \\
\hline elav $>$ DCr2, LIMK1 $1^{\text {RNAi(JF2063) }}$ & $36 \%(126)$ & $14 \%$ & $26 \%$ & $10 \%(121)$ & $1 \%$ & $9 \%$ \\
\hline elav $>$ DCr2, LIMK1 ${ }^{\text {RNAi(HM05159) }}$ & $46 \%(133)$ & $14 \%$ & $38 \%$ & $17 \%(121)$ & $0 \%$ & $17 \%$ \\
\hline $\operatorname{frac}^{\Delta 1} /+$ & $21 \%(160)$ & $1 \%$ & $19 \%$ & $6 \%(136)$ & $0 \%$ & $6 \%$ \\
\hline$L I M K 1^{E Y 08757} /+$ & $16 \%(107)$ & $1 \%$ & $15 \%$ & $6 \%(105)$ & $2 \%$ & $4 \%$ \\
\hline $\operatorname{LIMK1}^{\mathrm{EY} 08757} /_{+} ; \mathrm{frac}^{\Delta 1} /+$ & $27 \%(113)$ & $9 \%$ & $18 \%$ & $21 \%(114)$ & $6 \%$ & $16 \%$ \\
\hline $\mathrm{LIMK1}^{\mathrm{EY} 08757} ; \mathrm{frac}^{\Delta 1}$ & $58 \%(156)$ & $10 \%$ & $55 \%$ & $30 \%(42)$ & $2 \%$ & $28 \%$ \\
\hline
\end{tabular}

$n$, The number of hemisegments scored for each genotype.

${ }^{a}$ Hemisegments may have defects in both misprojection and hypofasciculation, yielding a total defasciculation that is not the sum of the two separate categories.

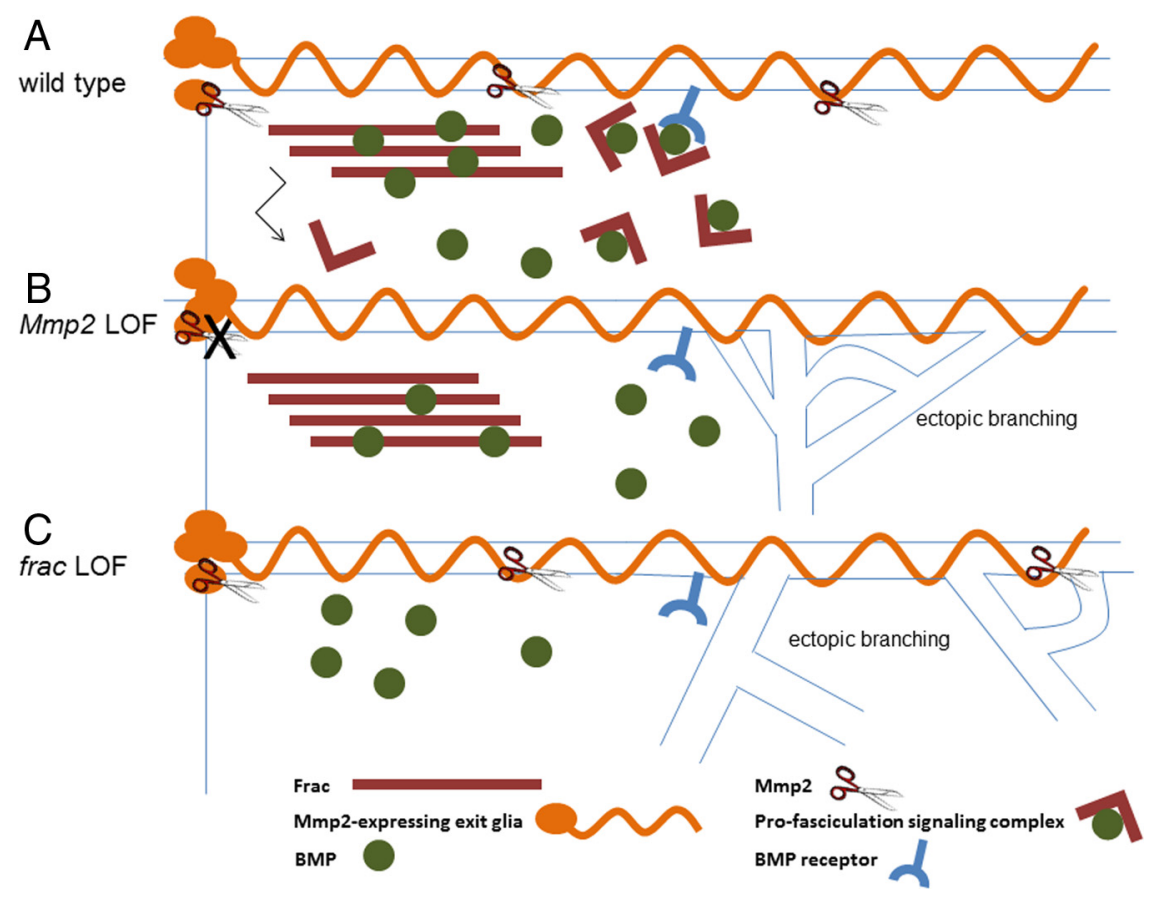

Figure 7. Model of the interaction between Mmp2 and Frac during axon guidance. $A$, In wild type, both full-length Frac and Frac protein fragments bind an BMP molecule. Cleaved Frac fragments form a signaling complex with available BMP and signal axons to stay bundled. $\boldsymbol{B}$, In Mmp2 LOF mutants, there is no protease to generate Frac fragments and therefore a signaling complex cannot form, resulting in increased defasciculation. $C$, In frac LOF mutants, there is no full-length or cleaved Frac present to bind the BMP molecule, preventing downstream signaling from occurring and resulting in increased defasciculation.

roles in limiting the bioavailability of TGF $\beta$ s (ten Dijke and Arthur, 2007; Ramirez and Rifkin, 2009). TGF $\beta$ s are targeted to Fibrillin scaffolds by latent TGF $\beta$-binding proteins and are released from this latent Fibrillin-associated complex via proteolysis and integrin-mediated activation. Support for the inhibitory role for Fibrillins in the TGF $\beta$ pathway comes from work on human MFS, which is a connective tissue disorder caused by mutations in human Fibrillin-1. Remarkably, the aortic root dilation associated with MFS can be attenuated or reversed with losartan, a drug with anti-TGF $\beta$ signaling activity (Habashi et al.,
2006; Brooke et al., 2008). The implication from these studies is that Fibrillin normally sequesters TGF $\beta$, so that a decrease in Fibrillin dosage results in excessive TGF $\beta$ signaling. If a related mechanism is at play in Mmp2-Frac signaling, we would predict that (1) frac LOF embryos would phenotypically resemble Mmp2 GOF because in both scenarios too much signal is released, and that (2) frac LOF embryos would exhibit the opposite phenotype of Mmp2 LOF because in the first case excess signal is released, whereas in Mmp2 LOF mutants, too little signal is generated. The finding that frac LOF and Mmp2 LOF mutants display essentially identical phenotypes, which are opposite to those displayed by Mmp2 GOF, suggests that Frac does not simply act to sequester or store a guidance cue.

A second model is that the Frac cleavage product has intrinsic signaling activity. Until recently, there have been few compelling examples of the direct action of matrix molecules in signaling in neuronal development. However, the Barres laboratory has demonstrated that Thrombospondin (TSP), another EGF-domaincontaining matrix protein, is secreted by astrocytes to promote synaptogenesis (Christopherson et al., 2005). They further demonstrated that EGF domains in TSP directly bind to the neuronal $\alpha 2 \delta$-1 calcium channel subunit to increase synapse formation (Eroglu et al., 2009). If Frac were solely responsible for providing the pro-fasciculation signal, the simplest prediction is that increasing Frac levels would increase axon bundling. In fact, we find that motor axons separate prematurely and inappropriately in $24 B>$ frac embryos, arguing against this model. We interpret the pathfinding phenotypes in these embryos to result from a "dominant negative-like" effect of elevated Frac. If the addition 
of excess Frac to the signaling system effectively dilutes out the guidance cue (Fig. 7, BMP), then decreased fasciculation is expected to result.

Frac is structurally related to the vertebrate Fibrillin family, making the interaction between Fibrillins and TGF $\beta$ s potentially relevant. Furthermore, in Drosophila, BMPs have been shown to be under complex regulation by type IV Collagens (Wang et al., 2008a), underscoring the essential role(s) matrix molecules play in this pathway. In addition, pMad is present in motoneuron nuclei as motor axons leave the ventral nerve cord (Marqués et al., 2002), demonstrating that the pathway is active during guidance. In light of these data, we analyzed genetic interactions between frac and the BMP pathway. Strikingly, activation of BMP signaling in motoneurons via overexpression of an activated type I receptor $\left(s a x^{a c t}\right)$ strongly suppresses the guidance defects associated with both frac LOF and GOF, arguing that frac regulates a BMP cue. This hypothesis is supported by motor axon guidance phenotypes displayed by LIMK1 mutants and genetic interactions between frac and LIMK1. In conclusion, we have described a novel pathway controlling motor axon targeting. We propose that Mmp2 expressed on the surface of exit glia controls the processing of the matrix molecule Frac, which sends a profasciculation signal to extending axons. These findings highlight the complex interactions between motor axons, glia, and the mesodermal matrix during motor axon targeting and lay the groundwork for future investigations into Mmp2-Frac pathway function.

\section{References}

Becker S, Pasca G, Strumpf D, Min L, Volk T (1997) Reciprocal signaling between Drosophila epidermal muscle attachment cells and their corresponding muscles. Development 124:2615-2622.

Bozdagi O, Nagy V, Kwei KT, Huntley GW (2007) In vivo roles for matrix metalloproteinase-9 in mature hippocampal synaptic physiology and plasticity. J Neurophysiol 98:334-344.

Brooke BS, Habashi JP, Judge DP, Patel N, Loeys B, Dietz HC 3rd (2008) Angiotensin II blockade and aortic-root dilation in Marfan's syndrome. N Engl J Med 358:2787-2795.

Charbonneau NL, Ono RN, Corson GM, Keene DR, Sakai LY (2004) Fine tuning of growth factor signals depends on fibrillin microfibril networks. Birth Defects Res C Embryo Today 72:37-50.

Chaudhry SS, Cain SA, Morgan A, Dallas SL, Shuttleworth CA, Kielty CM (2007) Fibrillin-1 regulates the bioavailability of TGFbeta1. J Cell Biol 176:355-367.

Christopherson KS, Ullian EM, Stokes CC, Mullowney CE, Hell JW, Agah A, Lawler J, Mosher DF, Bornstein P, Barres BA (2005) Thrombospondins are astrocyte-secreted proteins that promote CNS synaptogenesis. Cell 120:421-433.

Coleman HA, Labrador JP, Chance RK, Bashaw GJ (2010) The Adam family metalloprotease Kuzbanian regulates the cleavage of the roundabout receptor to control axon repulsion at the midline. Development 137:2417-2426.

Comer FI, Hart GW (2000) O-Glycosylation of nuclear and cytosolic proteins. Dynamic interplay between O-GlcNAc and O-phosphate. J Biol Chem 275:29179-29182.

Dickson BJ (2002) Molecular mechanisms of axon guidance. Science 298:1959-1964

Dietz HC, Loeys B, Carta L, Ramirez F (2005) Recent progress towards a molecular understanding of Marfan syndrome. Am J Med Genet C Semin Med Genet 139C:4-9.

Downing AK, Knott V, Werner JM, Cardy CM, Campbell ID, Handford PA (1996) Solution structure of a pair of calcium-binding epidermal growth factor-like domains: implications for the Marfan syndrome and other genetic disorders. Cell 85:597-605.

Durfee T, Becherer K, Chen PL, Yeh SH, Yang Y, Kilburn AE, Lee WH, Elledge SJ (1993) The retinoblastoma protein associates with the protein phosphatase type 1 catalytic subunit. Genes Dev 7:555-569.
Eaton BA, Davis GW (2005) LIM Kinase1 controls synaptic stability downstream of the type II BMP receptor. Neuron 47:695-708.

Eroglu C, Allen NJ, Susman MW, O'Rourke NA, Park CY, Ozkan E, Chakraborty C, Mulinyawe SB, Annis DS, Huberman AD, Green EM, Lawler J, Dolmetsch R, Garcia KC, Smith SJ, Luo ZD, Rosenthal A, Mosher DF, Barres BA (2009) Gabapentin receptor alpha2delta-1 is a neuronal thrombospondin receptor responsible for excitatory CNS synaptogenesis. Cell 139:380-392.

Fambrough D, Goodman CS (1996) The Drosophila beaten path gene encodes a novel secreted protein that regulates defasciculation at motor axon choice points. Cell 87:1049-1058.

Freeman MR (2006) Sculpting the nervous system: glial control of neuronal development. Curr Opin Neurobiol 16:119-125.

Habashi JP, Judge DP, Holm TM, Cohn RD, Loeys BL, Cooper TK, Myers L, Klein EC, Liu G, Calvi C, Podowski M, Neptune ER, Halushka MK, Bedja D, Gabrielson K, Rifkin DB, Carta L, Ramirez F, Huso DL, Dietz HC (2006) Losartan, an AT1 antagonist, prevents aortic aneurysm in a mouse model of Marfan syndrome. Science 312:117-121.

Haerry TE, Khalsa O, O'Connor MB, Wharton KA (1998) Synergistic signaling by two BMP ligands through the SAX and TKV receptors controls wing growth and patterning in Drosophila. Development 125:3977-3987.

Handford P, Downing AK, Rao Z, Hewett DR, Sykes BC, Kielty CM (1995) The calcium binding properties and molecular organization of epidermal growth factor-like domains in human fibrillin-1. J Biol Chem 270:6751-6756.

Hattori M, Osterfield M, Flanagan JG (2000) Regulated cleavage of a contact-mediated axon repellent. Science 289:1360-1365.

Hehr CL, Hocking JC, McFarlane S (2005) Matrix metalloproteinases are required for retinal ganglion cell axon guidance at select decision points. Development 132:3371-3379.

Hynes RO (2009) The extracellular matrix: not just pretty fibrils. Science 326:1216-1219.

Hynes RO, Zhao Q (2000) The evolution of cell adhesion. J Cell Biol 150:F89-F96.

James P, Halladay J, Craig EA (1996) Genomic libraries and a host strain designed for highly efficient two-hybrid selection in yeast. Genetics 144:1425-1436.

Kessenbrock K, Plaks V, Werb Z (2010) Matrix metalloproteinases: regulators of the tumor microenvironment. Cell 141:52-67.

Khalsa O, Yoon JW, Torres-Schumann S, Wharton KA (1998) TGF-beta/ BMP superfamily members, Gbb-60A and Dpp, cooperate to provide pattern information and establish cell identity in the Drosophila wing. Development 125:2723-2734.

Kiehart DP, Feghali R (1986) Cytoplasmic myosin from Drosophila melanogaster. J Cell Biol 103:1517-1525.

Llano E, Pendás AM, Aza-Blanc P, Kornberg TB, López-Otín C (2000) Dm1-MMP, a matrix metalloproteinase from Drosophila with a potential role in extracellular matrix remodeling during neural development. J Biol Chem 275:35978-35985.

Llano E, Adam G, Pendás AM, Quesada V, Sánchez LM, Santamariá I, Noselli S, López-Otín C (2002) Structural and enzymatic characterization of Drosophila Dm2-MMP, a membrane-bound matrix metalloproteinase with tissue-specific expression. J Biol Chem 277:23321-23329.

Marqués G, Bao H, Haerry TE, Shimell MJ, Duchek P, Zhang B, O’Connor MB (2002) The Drosophila BMP type II receptor Wishful Thinking regulates neuromuscular synapse morphology and function. Neuron 33:529-543.

Metaxakis A, Oehler S, Klinakis A, Savakis C (2005) Minos as a genetic and genomic tool in Drosophila melanogaster. Genetics 171:571-581.

Meyer F, Aberle H (2006) At the next stop sign turn right: the metalloprotease Tolloid-related 1 controls defasciculation of motor axons in Drosophila. Development 133:4035-4044.

Miller CM, Page-McCaw A, Broihier HT (2008) Matrix metalloproteinases promote motor axon fasciculation in the Drosophila embryo. Development 135:95-109.

Morin X, Daneman R, Zavortink M, Chia W (2001) A protein trap strategy to detect GFP-tagged proteins expressed from their endogenous loci in Drosophila. Proc Natl Acad Sci U S A 98:15050-15055.

Mumm JS, Kopan R (2000) Notch signaling: from the outside in. Dev Biol 228:151-165.

Neptune ER, Frischmeyer PA, Arking DE, Myers L, Bunton TE, Gayraud B, Ramirez F, Sakai LY, Dietz HC (2003) Dysregulation of TGF-beta acti- 
vation contributes to pathogenesis in Marfan syndrome. Nat Genet 33:407-411.

Page-McCaw A, Serano J, Santé JM, Rubin GM (2003) Drosophila matrix metalloproteinases are required for tissue remodeling, but not embryonic development. Dev Cell 4:95-106.

Page-McCaw A, Ewald AJ, Werb Z (2007) Matrix metalloproteinases and the regulation of tissue remodelling. Nat Rev Mol Cell Biol 8:221-233.

Parker L, Ellis JE, Nguyen MQ, Arora K (2006) The divergent TGF-beta ligand Dawdle utilizes an activin pathway to influence axon guidance in Drosophila. Development 133:4981-4991.

Ramirez F, Dietz HC (2007) Fibrillin-rich microfibrils: structural determinants of morphogenetic and homeostatic events. J Cell Physiol $213: 326-330$

Ramirez F, Rifkin DB (2009) Extracellular microfibrils: contextual platforms for TGFbeta and BMP signaling. Curr Opin Cell Biol 21:616-622.

Rodríguez D, Morrison CJ, Overall CM (2010) Matrix metalloproteinases: what do they not do? New substrates and biological roles identified by murine models and proteomics. Biochim Biophys Acta 1803:39-54.

Sakai LY, Keene DR, Engvall E (1986) Fibrillin, a new 350-kD glycoprotein, is a component of extracellular microfibrils. J Cell Biol 103:2499-2509.

Sengle G, Charbonneau NL, Ono RN, Sasaki T, Alvarez J, Keene DR, Bächinger HP, Sakai LY (2008) Targeting of bone morphogenetic protein growth factor complexes to fibrillin. J Biol Chem 283:13874-13888.

Sepp KJ, Auld VJ (2003) Reciprocal interactions between neurons and glia are required for Drosophila peripheral nervous system development. J Neurosci 23:8221-8230.

Sepp KJ, Schulte J, Auld VJ (2001) Peripheral glia direct axon guidance across the CNS/PNS transition zone. Dev Biol 238:47-63.

Serpe M, O'Connor MB (2006) The metalloprotease tolloid-related and its TGF-beta-like substrate Dawdle regulate Drosophila motoneuron axon guidance. Development 133:4969-4979.

Sink H, Rehm EJ, Richstone L, Bulls YM, Goodman CS (2001) sidestep encodes a target-derived attractant essential for motor axon guidance in Drosophila. Cell 105:57-67.

Strumpf D, Volk T (1998) Kakapo, a novel cytoskeletal-associated protein is essential for the restricted localization of the neuregulin-like factor, vein, at the muscle-tendon junction site. J Cell Biol 143:1259-1270.

ten Dijke P, Arthur HM (2007) Extracellular control of TGFbeta signalling in vascular development and disease. Nat Rev Mol Cell Biol 8:857-869.

Terman JR, Kolodkin AL (2004) Nervy links protein kinase a to plexinmediated semaphorin repulsion. Science 303:1204-1207.
Urbano JM, Torgler CN, Molnar C, Tepass U, López-Varea A, Brown NH, de Celis JF, Martín-Bermudo MD (2009) Drosophila laminins act as key regulators of basement membrane assembly and morphogenesis. Development 136:4165-4176.

Vallejo AN, Pogulis RJ, Pease LR (1994) In vitro synthesis of novel genes: mutagenesis and recombination by PCR. PCR Methods Appl 4:S123-S130.

Vactor DV, Sink H, Fambrough D, Tsoo R, Goodman CS (1993) Genes that control neuromuscular specificity in Drosophila. Cell 73:1137-1153.

Wang X, Harris RE, Bayston LJ, Ashe HL (2008a) Type IV collagens regulate BMP signalling in Drosophila. Nature 455:72-77.

Wang XB, Bozdagi O, Nikitczuk JS, Zhai ZW, Zhou Q, Huntley GW (2008b) Extracellular proteolysis by matrix metalloproteinase- 9 drives dendritic spine enlargement and long-term potentiation coordinately. Proc Natl Acad Sci U S A 105:19520-19525.

Wharton KA, Cook JM, Torres-Schumann S, de Castro K, Borod E, Phillips DA (1999) Genetic analysis of the bone morphogenetic protein-related gene, gbb, identifies multiple requirements during Drosophila development. Genetics 152:629-640.

Winberg ML, Noordermeer JN, Tamagnone L, Comoglio PM, Spriggs MK, Tessier-Lavigne M, Goodman CS (1998) Plexin A is a neuronal semaphorin receptor that controls axon guidance. Cell 95:903-916.

Yasothornsrikul S, Davis WJ, Cramer G, Kimbrell DA, Dearolf CR (1997) viking: identification and characterization of a second type IV collagen in Drosophila. Gene 198:17-25.

Yasunaga K, Kanamori T, Morikawa R, Suzuki E, Emoto K (2010) Dendrite reshaping of adult Drosophila sensory neurons requires matrix metalloproteinase-mediated modification of the basement membranes. Dev Cell 18:621-632.

Yu HH, Araj HH, Ralls SA, Kolodkin AL (1998) The transmembrane Semaphorin Sema I is required in Drosophila for embryonic motor and CNS axon guidance. Neuron 20:207-220.

Yu HH, Huang AS, Kolodkin AL (2000) Semaphorin-1a acts in concert with the cell adhesion molecules fasciclin II and connectin to regulate axon fasciculation in Drosophila. Genetics 156:723-731.

Zhang H, Hu W, Ramirez F (1995) Developmental expression of fibrillin genes suggests heterogeneity of extracellular microfibrils. J Cell Biol 129:1165-1176.

Zhang S, Dailey GM, Kwan E, Glasheen BM, Sroga GE, Page-McCaw A (2006) An MMP liberates the Ninjurin A ectodomain to signal a loss of cell adhesion. Genes Dev 20:1899-1910. 